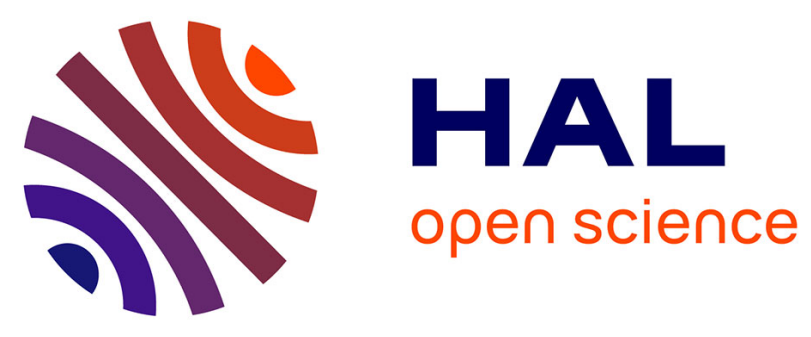

\title{
Tuning up Mind's Pattern to Nature's Own Idea: Eddington's Early Twenties Case for Variational Derivatives
}

Ivahn Smadja

\section{- To cite this version:}

Ivahn Smadja. Tuning up Mind's Pattern to Nature's Own Idea: Eddington's Early Twenties Case for Variational Derivatives. Studies in History and Philosophy of Science Part B: Studies in History and Philosophy of Modern Physics, 2010, 41 (2), pp.128-145. 10.1016/j.shpsb.2010.03.001 . halshs$00456369 \mathrm{v} 2$

\section{HAL Id: halshs-00456369 \\ https://shs.hal.science/halshs-00456369v2}

Submitted on 28 Feb 2010

HAL is a multi-disciplinary open access archive for the deposit and dissemination of scientific research documents, whether they are published or not. The documents may come from teaching and research institutions in France or abroad, or from public or private research centers.
L'archive ouverte pluridisciplinaire HAL, est destinée au dépôt et à la diffusion de documents scientifiques de niveau recherche, publiés ou non, émanant des établissements d'enseignement et de recherche français ou étrangers, des laboratoires publics ou privés. 


\title{
Tuning up Mind's Pattern to Nature's Own Idea : Eddington's Early Twenties Case for Variational Derivatives ${ }^{\text {th }}$
}

\author{
Ivahn Smadja \\ Université Paris Diderot - Paris 7 - CNRS UMR 7219
}

\begin{abstract}
This paper sets out to show how Eddington's early twenties case for variational derivatives significantly bears witness to a steady and consistent shift in focus from a resolute striving for objectivity towards "selective subjectivism" and structuralism. While framing his so-called "Hamiltonian derivatives" along the lines of previously available variational methods allowing to derive gravitational field equations from an action principle, Eddington assigned them a theoretical function of his own devising in The Mathematical Theory of Relativity (1923). I make clear that two stages should be marked out in Eddington's train of thought if the meaning of such variational derivatives is to be adequately assessed. As far as they were originally intended to embody the mind's collusion with nature by linking atomicity of matter with atomicity of action, variational derivatives were at first assigned a dual role requiring of them not only to express mind's craving for permanence but also to tune up mind's privileged pattern to "Nature's own idea". Whereas at a later stage, as affine field theory would provide a framework for world-building, such "Hamiltonian differentiation" would grow out of tune through gauge-invariance and, by disregarding how mathematical theory might precisely come into contact with actual world, would be turned into a mere heuristic device for structural knowledge.
\end{abstract}

Key words: general relativity, Hamilton's principle, action, gauge invariance, structuralism, Eddington, Einstein, Hilbert, Weyl

\section{Introduction}

Eddington's philosophical train of thought in the twenties may be outlined as a steady and consistent shift in focus from a resolute striving for objectivity towards "selective subjectivism" and structuralism. As a consequence of the identification of phenomenological matter with a well-behaved tensor, pattern recognition would appear as taking precedence over knowledge of the external world as such, inasmuch as the mind would allegedly single out a specific pattern as permanent substance. While addressing the challenging issue whether the unified scheme in which the laws of physics had been so

\footnotetext{
Submitted : May 1, 2009. Revised : November 3, 2009. Accepted : February 23, 2010. 
far successfully woven would wholly stem from the mind, as his current views seemed to suggest strongly in the early twenties, Eddington however inclined to admit that "there are laws which appear to have seat in external nature" ${ }^{1}$ and to acknowledge the law of atomicity as probably the only genuine law of nature. Since matter happens to exist only in certain lumps corresponding to atoms or electrons, the inescapable question, as eloquently raised by Eddington, would thus be : "whence arises this discontinuity?" 2 . Insofar as the mind mostly endeavours to "smooth the discontinuities of nature into continuous perception" ${ }^{3}$, there is little plausibility in ascribing atomicity of matter to the workings of the mind, so that the cause thereof might rather be something in the nature of things themselves, whereas the analysis into point-events might not be ultimate. Supposing that a further analysis could delve deeper into the atomic structure of matter so that something still more fundamental could be attained, laws of nature such as atomicity would eventually be accounted for as identities. But, as Eddington points out, "this more ultimate analysis stands on a different plane from that by which the point-events were reached" 4 . How then could these two different scales, namely molecular structure of matter versus continuous structure of matter, could be brought into one single, even if provisional, scheme? Eddington suggests some kind of trade-off between microscopic and macroscopic levels of reality. Though, strictly speaking, the regions lying between the electrons in which matter is scattered are not to be thought of as curved, while the regions inside the electrons should be held outright cut out of space-time, all that is required in macroscopic physics is to know "not the actual values of the $g$ 's at a point, but their average values through a region, (...) [so that $]$ uncurved space-time studded with holes is replaced by an equivalent fully curved space-time without holes" ${ }^{5}$. Though generalizing previously well-known variational techniques allowing to derive field equations from an action principle, Eddington originally devised his "Hamiltonian derivatives" 6 as the appropriate mathematical apparatus allowing to implement this equivalence by conciliating apparently contradictory requirements. Both molecular and continuous schemes should indeed be conjoined, though kept distinct, as embodying part of the physical truth, since the space-time interval can be seen as "an average summary which suffices for our coarse methods of investigation and holds true [even if] only statistically" 7 , so that, as Eddington avers, it is preferable to think of matter and energy, "not as agents causing the degrees of curvature of the world, but as parts of our perceptions of the existence of the curvature" 8 .

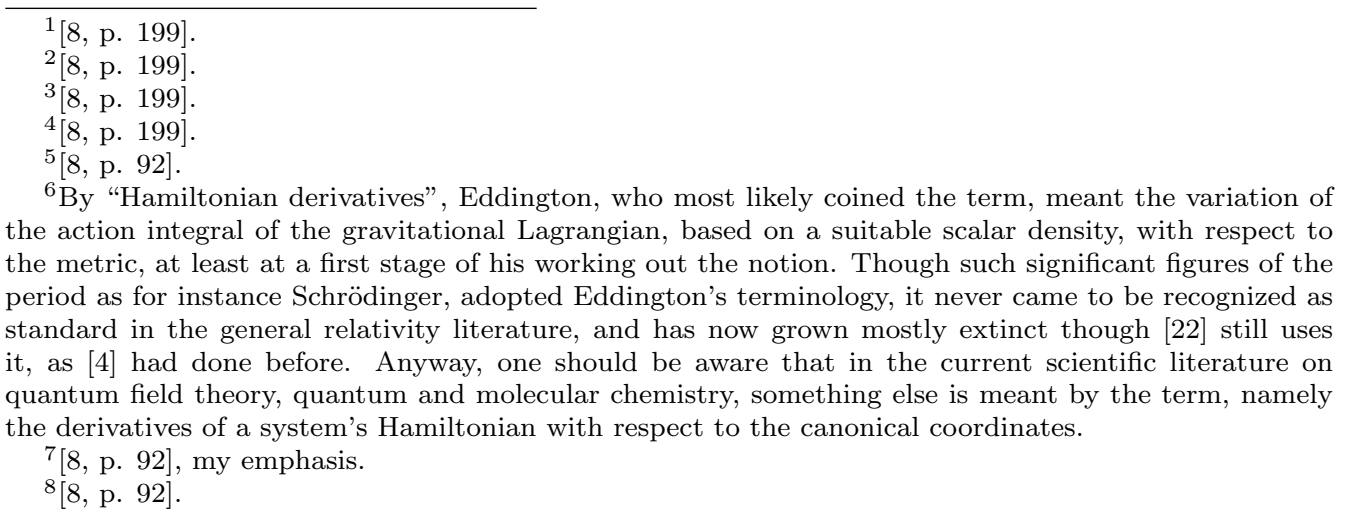


So as to make clear why Eddington may have been led to envision "Hamiltonian differentiation" as a way out of the predicament consisting in such alternative levels of physical reality being apparently irreconcilable, it may prove useful to outline the animated debate that opposed, a few years before Eddington's own attempts, some of the main protagonists engaged in the pursuit of a unified field theory, concerning whether, how and to what extent a variational derivation of gravitational field equations should be deemed effective and legitimate. As is well known, Hilbert and Einstein arrived at gravitational field equations by two different routes though both eventually agreeing on a common standard formulation during the dramatic weeks of November, 1915. The way the interactions and exchanges that occurred between them in this short period of time may have affected their respective approaches and somewhat deflected their ultimate contributions has been extensively studied in the recent years (cf. [36], [33], [7], [37], [27]). The timeline should be kept in mind here if a clear understanding of both positions is to be gained. From June 29 to July 7, 1915, Einstein visited Hilbert in Göttingen and, later commenting on this meeting, would state that he "he had the great pleasure of seeing that everything [meaning presumably, as explained by [7, p. 321], the equivalence of inertial and gravitational effects as being susceptible of an expression in terms of the metric tensor] was understood down to the details" ${ }^{9}$. A few months later, on the base of previous work on the structure of matter, Hilbert presented two communications on "the foundations of physics" in Göttingen circles, first on November 16, then four days later on November 20, 1915. Though invited to attend these events, Einstein declined since at the same time he was working hard on the correct computation of the perihelion advance of Mercury as predicted by his new field equations. Extant galley proofs of Hilbert's Göttingen presentation, dated December 6, 1915, bearing the author's handwritten annotation "Erste Korrektur meiner ersten Note", have been carefully compared to the published version, viz. [23], dated March 16, 1916. Though incomplete, these proofs help figuring out how Hilbert's line of thought evolved during these crucial months from a derivation of the yet correct gravitational field equations of general relativity - though very likely (since the decisive passage in this respect is missing) only in implicit form - in terms of a variational principle applied to a Lagrangian conceived as a sum of a gravitational part and a matter part on the one hand, to an ultimate incorporation and homologation of Einstein's tensor within his own peculiar scheme in the printed version on the other hand (cf. [37]). In the meantime, Einstein who had struggled to master general covariance after having disposed of the objection relating to the "hole argument" that had blocked him during the previous months ${ }^{10}$, gave a series of four communications to the Berlin Academy culminating on November 25, 1915, with the presentation of the final version of his generally covariant gravitational field equations. On the base of his hearing about Hilbert's Göttingen talks, Einstein would complain, without though naming anyone, in a oft-quoted letter to Zangger ${ }^{11}$, dated November 26, 1915, about some attempts aiming at "nostrifying" 12 his theory. However intricate such crossed influences

\footnotetext{
${ }^{9}$ Einstein to Sommerfeld, July 15, 1915, [18, Doc. 96], our emphasis

${ }^{10}$ As pointed out by [7, p. 347], Einstein eventually put aside the "hole argument" by convincing himself that "nothing is real physically except for the entirety of the spatio-temporal point coincidences" (Einstein to Besso, January 3, 1916, [18, Doc. 178], Italics in the original).

11 [18, Doc. 152].

${ }^{12}$ Cf. [7, p. 419-422] for an explanation of what "nostrification" as a key feature of Göttingen culture, means.
} 
may have been, one major difference between Hilbert's and Einstein's view of the way gravitational field equations should be obtained remains of concern if Eddington's later work is to be correctly assessed, namely their disagreement on the status and the role of variational methods.

Hilbert favoured an axiomatic approach to the foundations of physics by which he meant a grand theory concerning physics in general that would mainly solve the problem of the structure of matter which had been his longstanding central focus of interest and with respect to which gravitational field equations only appeared as a secondary and almost incidental goal. More specifically, Hilbert's 1915 axiomatization of physics aimed at combining in a broad integrated whole Mie's electromagnetic theory of matter with some of the ideas Einstein had presented in Göttingen during the previous summer term, essentially the principle of equivalence and general covariance. Mie's 1912 theory ([28]) set out to substantiate the view that the electron has no independent physical existence apart from the ether, so that what is given to our perception as material particles would be nothing more than singularities of the ether ${ }^{13}$. Whereas the field of a charged particle would presumably grow infinite inside the particle according to Coulomb's law, as [7] explains, "Mie's equations generalized those of Maxwell's theory in such a way that the repulsive forces predicted inside the electron would be compensated by other forces, of purely electrical nature, as well. Moreover, outside the electron, the deviation of Mie's equations from Maxwell's becomes undetectable" ${ }^{14}$. Inasmuch as he thought of electric and gravitational actions as being derived from the very same forces that are assumed by his theory of matter, Mie tried to develop new Lorentz covariant equations that would account not only for the phenomena that take place between the material particles but also for those that take place inside them.

Guided by what Klein would later stigmatize as a "fanatical" ${ }^{15}$ belief in the power of variational methods as a means to disclose the hidden laws of nature, Hilbert conceived of the axiomatization of any particular physical discipline as requiring that a general variational argument be supplemented by specific axioms "meant to capture the essence of the theory in question"16, and thus incorporated Mie's theory of matter in the form of such a variational principle as the central axiom of his own theory. Assuming a spacetime manifold with coordinates $w_{s}, s=1,2,3,4$, Hilbert considers the ten gravitational potentials $g_{\mu \nu}$ acknowledged as "first introduced by Einstein" 17 and four electrodynamic potentials $q_{s}$ which, unlike the former, though introduced on a equal footing with them, lack any transparent geometrical interpretation and "appear in the theory as somewhat external elements" ${ }^{18}$. The key idea is then to derive field equations by a variational principle from a Hamiltonian function $H$, a "world-function", of these $g_{\mu \nu}$ with their

\footnotetext{
${ }^{13}$ Cf. [7, p. 300] : "[according to Mie $]$ the electric and the magnetic fields are present inside the electron as well. This expresses the assumption that the electrons are an organic part of the ether, rather than foreign elements added to it, as was the common belief among certain physicists at the time. The electron is thus conceived as a non-sharply delimited, highly dense, nucleus in the ether that extends continually and infinitely into an atmosphere of electrical charge. An atom is a concentration of electrons, and the high intensity of the electric field around it is what should ultimately explain the phenomenon of gravitation."

14 [7, p. 300].

${ }^{15}$ Klein to Pauli, May 8, 1921, quoted by [7, p. 436].

${ }^{16}[7$, p. 333].

$17[23$, p. 395]

18 [41, p. 57].
} 
first and second derivatives $\frac{\partial g_{\mu \nu}}{\partial w_{l}}, \frac{\partial^{2} g_{\mu \nu}}{\partial w_{l} \partial w_{k}}$, and the $q_{s}$ with their first derivatives $\frac{\partial q_{q}}{\partial w_{l}}$. Whereas axiom I, viz. "Mie's axiom of the world-function", states that under infinitesimal variations of the fourteen potentials $g_{\mu \nu}, q_{s}$, the variation of the action integral vanishes,

$$
\delta \int H \sqrt{g} d \omega=0
$$

axiom II, viz. "the axiom of general invariance", ensures that $H$ is invariant with respect to any of the transformation of the coordinates $w_{s}$. Among the Euler-Lagrange equations yielded by the variational principle, Hilbert identifies those associated with the variation of the $g_{\mu \nu}$ with the ten gravitational field equations and those associated with the variation of the $q_{s}$ with the four electrodynamic ones, respectively denoted in abbreviated form

$$
[\sqrt{g} H]_{\mu \nu}=0, \quad[\sqrt{g} H]_{h}=0 .
$$

A mathematical theorem (viz. Theorem I) which Hilbert presented as the "Leitmotiv for the construction of [the] theory" 19 and which appears with hindsight as boiling down to Noether's second theorem, then presumably allowed for unification of gravitational and electromagnetic fields, insofar as the original fourteen Euler-Lagrange equations are proven to reduce to only ten independent ones. Hilbert thence claimed that "the electrodynamic phenomena are an effect of gravitation" ${ }^{20}$ inasmuch as he erroneously inferred that the electrodynamic equations $[\sqrt{g H}]_{h}=0$ were a mere consequence of the gravitational ones $[\sqrt{g H}]_{\mu \nu}=0$, the latter being tacitly identified as a whole with the independent equations. Though generally covariant and as such very much akin to the equations Einstein obtained at about the same time, these gravitational field equations did not yet constitute the fundamental equations of physics in Hilbert's view, since four supplementary non generally covariant energy equations were then required so as to restore causality ${ }^{21}$. Within such an axiomatic setting, Hilbert still worked out the theory further by postulating that the Hamiltonian function be of a certain form, namely that it should be composed of a gravitational and an electromagnetic part

$$
H=K+L
$$

where $K$ is the scalar curvature whereas $L$ remains unspecified besides being assumedly dependent, for the sake of simplicity, on the sole gravitational potentials $g_{\mu \nu}$ and the electrodynamic potential $q_{s}$ and their first derivatives $\frac{\partial q_{q}}{\partial w_{l}}$. The Euler-Lagrange equations associated with the variation of the $g_{\mu \nu}$ then provide fine-grained gravitational field equations

$$
[\sqrt{g} K]_{\mu \nu}+\frac{\partial \sqrt{g} L}{\partial g^{\mu \nu}}=0
$$

\footnotetext{
19 [23, p. 396].

$20[23$, p. 397]

${ }^{21}$ In this respect, Einstein and Hilbert went reverse ways, insofar as the former doggedly strove for general covariance while not taking it for granted, whereas the latter was eventually led to counterbalance the so-called "axiom of general invariance" by the need to guarantee determinism for the equations of physics. Cf. [3] for an overall assessment of the epistemological significance of the axiomatic setting in which Hilbert addressed the problem raised by the tension between general covariance on the one hand, taken as a "an ideal regulative principle in the sense of Kant" ([3, p. 145]) which would condition objectivity, and causality on the other hand, conceived of by contrast as "a necessary condition imposed by the mind in structuring experience" ([3, p. 146]).
} 
where the second term on the left-hand side obtained by taking the variation of the metric tensor in the electromagnetic part of the action integral is none other than Mie's electromagnetic energy tensor, which, as Hilbert stressed it, "indicated [to him] the close connection between Einstein's general theory of relativity and Mie's electrodynamics and provided $[\mathrm{him}]$ with the conviction of the correctness of the theory developed here" ${ }^{22}$. Still in the proofs of the November communication, the gravitational field equations only appear in the implicit form given above (equ.(4)), whereas in the printed version, Hilbert gave them an explicit formulation

$$
[\sqrt{g} K]_{\mu \nu}=\sqrt{g}\left(K_{\mu \nu}-\frac{1}{2} K g_{\mu \nu}\right)
$$

which he claimed to follow "easily and without calculations" 23 from the implicit form, by simply pointing out that " $K_{\mu \nu}$ is the only second rank tensor, besides $g_{\mu \nu}$, and $K$ is the only invariant that can be made up only with the $g^{\mu \nu}$ and their first and second derivatives $g_{k}^{\mu \nu}, g_{k l}^{\mu \nu} .{ }^{24}$ Though this justificatory argument seems to have played an important role in the framing of the received gravitational field equations ${ }^{25}$ since both Einstein and Hilbert used it, Eddington would later circumvent it by considering alternative Lagrangians giving rise to higher order gravitational field equations as equally acceptable (Cf. $\S 3.2$ below). Whereas Einstein's explicit articulation, occurring in the meantime, of the gravitational field equations may have induced Hilbert in making them explicit as well in his own later written version, thus gaining extra support from convergence with Einstein's formulation, Hilbert's approach still remains utterly different from Einstein's and can be seen as the "culmination of a research program" 26 whose main feature consists in combining axiomatization and variational methods with deep concern for the structure of matter, as the conclusion of the published version bears witness :

... not only our conceptions of space, time and motion have been modified in the direction suggested by Einstein, but I am also convinced that starting from the basic equations established here, the innermost - and so far concealed processes occurring inside the atom will be finally illuminated. ${ }^{27}$

Einstein distanced himself from Hilbert's view first by denying any alleged "close connection" between his gravitational field equations and Mie's theory of matter, and second by pointing out a presumably important weakness from the physical point of view in Hilbert's derivation of such field equations from a variational principle :

\footnotetext{
${ }^{22}[23$, p. 404]. Cf. [41, p. 59] for a comment on this passage.

23 [23, p. 405].

$24[23$, p. 405]

${ }^{25}$ The history of this justificatory argument is outlined in [7, p. 359] : "It seems that this kind of argument was first suggested by Grossmann to Einstein at the beginning of their collaboration. However, it was not until 1917 that Klein raised doubts about its validity and, at any rate, he was not able to find at that time any published proof of it. On Klein's request, Hermann Vermeil (1889-1959) by then his assistant, came up with a proof that, indeed, $K$ is the only scalar invariant involving linear combinations of $g^{\mu \nu}$ and its first and second derivatives. The proof itself was rather straightforward, but it required some effort. Later on, additional proofs were given by Weyl and Laue who also showed their precise connection with Einstein's argument."

$26[7$, p. 343].

$27[23$, p. 407], the translation is Leo Corry's ([7, p. 343]) and the emphasis is mine.
} 
The difficulty was not in finding the generally covariant equations from the $g_{\mu \nu}$; for this is easily achieved with the aid of the Riemann's tensor. Rather, it was hard to recognize that these equations are a generalization, that is, a simple and natural generalization of Newton's law. It has just been in the last few weeks that I succeeded in this ... whereas 3 years ago with my friend Grossmann, I had already taken into consideration the only possible generally covariant equations, which have now been shown to be the correct ones. ${ }^{28}$

Einstein did not dismiss Hamilton's principle as such as an acceptable way to derive field equations. Ever since [19], he had opted for a straightforward recourse to a variational derivation operating on an action integral akin to Hilbert's ${ }^{29}$ and would later go back to such methods ([15]) without though making any strong assumption on the constitution of matter, as Hilbert did. Therefore what was incriminated in his criticism was rather Hilbert's failure to convincingly provide, through variational methods, an overall encompassing theory of matter in the line of Mie, as he clearly articulated in a letter to Weyl, dated November 23, 1916.

Hilbert's assumption about matter appears childish to me, in the sense of a child who does not know of any of the tricks of the world outside. I am searching in vain for a physical indication that the Hamiltonian function for matter can be formed from the $\phi_{\nu}$ 's [viz. the electromagnetic potentials] without differentiation. At all events, mixing the solid considerations originating from the relativity postulate with such bold, unfounded hypotheses about the structure of the electron or matter cannot be sanctioned. I gladly admit that the search for a suitable hypothesis, or for the Hamiltonian function for the structural make-up of the electron, is one of the most important tasks of theory today. The "axiomatic method" can be of little use here, though. ${ }^{30}$

In about the same vein, Weyl would later emphasize "the maze of experimental facts which the physicist has to take into account [being] too manifold, their expansion too fast, and their aspect and relative weight too changeable for the axiomatic method to find a firm enough foothold, except in the thoroughly consolidated parts of our physical knowledge", thus favouring Einstein's way of "grop[ing his] way in the dark" as more appropriate to physical theory inasmuch as requiring "another type of experience and imagination than those of the mathematician" 31 . More specifically, Weyl's criticism focused on Hilbert's Hamiltonian function involving "still much too much arbitrariness [which] subsequent attempts (by Weyl, Eddington, Einstein himself and others) aimed to reduce" 32 . Whereas, by tracking down the last remnant of abstract empty thinking in Riemannian geometry, Weyl's own gauge fundamental concept (cf below $§ 4.1$ ) provided

\footnotetext{
${ }^{28}$ Einstein to Hilbert, November 18, 1915, [18, Doc. 148].

${ }^{29}$ In this respect, [7, p. 295-296] points out a significant fact indicating that Einstein may have originally borrowed the very idea of a variational derivation of gravitational field equations from Hilbert's circle : "It is ... remarkable that in a footnote to the article containing the variational derivation, Grossmann and Einstein thanked Paul Bernays for having suggested this very possibility for obtaining the equations. ... one wonders to what extent this enthusiasm for variational methods was not a direct result of the ever-present centrality of such techniques in the day-to-day routine of Hilbert and his circle."

${ }^{30}$ Einstein to Weyl, November 23, 1916, [18, Doc. 278], emphasis in the original.

31 [46, p. 653$]$

$32[46$, p. 654$]$
} 
an intertwining of gravitational and electromagnetic fields that would allegedly be more in agreement with experimental facts than mere Göttingen variational virtuosity, still Weyl himself persistently claimed that "the whole of physical phenomena may be derived from one single world-law of mathematical simplicity" ${ }^{33}$, namely from a suitable action principle, whatever be the difficulties, stressed by [21], that were to be encountered so as to find "a satisfactory Lagrangian from which the field equations of Weyl's theory can be derived" 34 . Reversely, by contrast with Einstein whose well-known objection to Weyl's theory did not prevent him though from deeming it a valuable attempt ${ }^{35}$. Hilbert would later judge it rather harshly as ill-conceived immoderate idealizing for which he coined the term "Hegelian physics" ${ }^{36}$, though claiming at the same time the paternity for most of the building blocks that Weyl used :

Having returned from Bucharest I have looked more carefully at the proofs of your book [viz. [43]] which gave me great pleasure, especially also the refreshing and enthusiastic presentation. I noticed that you did not even mention my first Göttingen note from 1915 even though the foundations of the gravitational theory, in particular the use of the Riemannian curvature in the Hamiltonian integral which you present on page 191, stems from me alone, as does the separation of the Hamiltonian function in $H-L$, the derivation of the Maxwellian equations, etc. Also the whole presentation of Mie's theory is precisely that which I gave for the first time in my first note on the foundations of physics. For Einstein's earlier work on his definitive theory of gravitation appeared at the same time as mine (namely in November 1915); Einstein's other papers, in particular on electrodynamics and on Hamilton's principle appeared however much later. ${ }^{37}$

In this context, Eddington's use of Hamilton's principle so as to derive field equations are to be demarcated from both Hilbert's and Weyl's, inasmuch as presumably tracing a different path toward unified field theory ${ }^{38}$. As is well known, Eddington suggested putting aside the metric as the fundamental concept and starting out right away with a suitable connection in terms of which a so-called "affine geometry" can be framed out. Unlike Weyl, Eddington hence distinguished this affine geometry as the "geometry of the world structure" from Riemannian geometry as the "natural geometry of the world", the latter being "the single true geometry in the sense understood by the physicist" whereas

\footnotetext{
$33[42$, p. 385 , footnote 4$]$.

34 [21, p. 35].

${ }^{35}$ Einstein indeed admired the "stroke of genius of first rank" ([18, Vol. 8B, Doc. 498, 710]), whereas decisively pointing out that since the measurements are made with infinitesimally small rigid rods and clocks, there should be no indeterminacy in the metric as would presumably be the case with Weyl's gauge idea.

$36[24$, p. 71 and 100]. By analogy with Hegel's stance who pretended to justify the number of planets, thus incorporating even contingent features of the world in his overall rational scheme, Weyl's theory was, in Hilbert's view, an instance of such theories aiming at deriving mathematically from general laws the values of all significant quantities in nature, given fundamental equations and fully articulated initial conditions.

${ }^{37}$ Hilbert to Weyl, April, 22, 1918, quoted in [33, p. 66].

${ }^{38}$ It should be noted that Eddington refers to the works of Mie (1912) and Hilbert (1915 and 1917) in the bibliography at the end of $[12$, p. 264], as "most likely to be helpful on particular points, or as of importance in the historic development". His use of an action principle is thus to be assessed with reference to these previous conceptions.
} 
the former is to be thought of as "the pure geometry applicable to a conceptual graphical representation of all the quantities concerned in physics" ${ }^{39}$. While we are accustomed to use graphs to represent geometrical or mechanical quantities, whether such graphs be naturally suggested, as for measured distances, directions, areas and volumes, or require convention, as for potentials, temperatures, forces, densities, etc., "we do not always draw the graphs on a sheet of paper" and graphical representation may also occur "in a conceptual mathematical space of any number of dimensions and possibly non-Euclidean geometry" ${ }^{40}$ such as affine geometry, while remaining entirely free from assumptions regarding the ultimate nature of things. But accordingly, unlike Hilbert, Eddington did not seek to incorporate from the outset a theory of the structure of matter, such as Mie's, in his variational scheme, though as noted by [21, p. 46], his motivation for affine field theory went beyond unification of gravitation and electromagnetism, and did not exclude to shed light on the problem of the electron :

In passing beyond Euclidean geometry, gravitation makes its appearance; in passing beyond Riemannian geometry, electromagnetic force appears; what remains to be gained by further generalization? Clearly, the non-Maxwellian binding forces which hold together an electron. But the problem of the electron must be difficult, and I cannot say whether the present generalization succeeds in providing the material for its solution. ${ }^{41}$

While embracing for a couple of years Eddington's affine field theory ${ }^{42}$, Einstein would endeavour, though eventually in vain, to tackle the problems relating to the electron within an affine framework, thus winding up with equations that Hilbert would later consider a return to his own 1915 results, even if "on a colossal detour via Levi-Civita, Weyl, Schouten, Eddington" ${ }^{43}$. However, in Eddington's original view, the nature of the electron seen as "a region of abnormal [viz. abnormally large] curvature" ${ }^{4}$ was thought of as an open problem undetermined by the theory as it were. Whereas acknowledging Eddington's distinction between "world geometry" and "natural geometry", Pauli for instance, as recalled by [21], conceded the union of gravitational and electromagnetic fields as only a phenomenological one, involving as such no recourse to the nature of the charged elementary particles ${ }^{45}$.

Eddington's case for variational derivatives hence originally appeared as an ingenious way to keep mathematical theory in step with physical reality while refraining from making any assumption on the nature of things. Supposing indeed atomicity to be

\footnotetext{
$39[12$, p. 198$]$

${ }^{40}[12$, p. 196$]$.

41 [11, p. 104], quoted and commented in [21, p. 46].

${ }^{42}$ Einstein thus wrote to Bohr, on January, 11, 1923 : "I believe I have finally understood the connection between electricity and gravitation. Eddington has come closer to the truth than Weyl", as quoted and commented in [21, p. 48].

${ }^{43}$ Cf. [27] for a comment on this quote from Hilbert's 1923 Hamburg Lectures on the foundations of physics.

${ }^{44}$ Cf. [21, p. 48].

${ }^{45}$ Cf. Pauli to Eddington, September, 20, 1923, [32, p. 115] : "I now do not at all believe that the problem of elementary particles can be solved by any theory applying the concept of continuously varying field strengths which satisfy certain differential equations to regions in the interior of elementary particles."
} 
a law inherent in nature, why should it be particularly concerned with matter, while quantum theory would rather focus on action? Inasmuch as action is deemed "the most fundamental thing in the real world of physics, although the mind passes it because of its lack of permanence" 46 , "Hamiltonian derivatives", as Eddington conceived of them at first, would thus ensure the mind's collusion with nature by linking atomicity of matter with atomicity of action. Whereas at a later stage, as affine field theory would provide a framework for graphical representation, the whole machinery of "Hamiltonian differentiation", enlarged so as to accommodate gauge invariance, would no more keep the mind in step with external reality and, by disregarding how mathematical theory might precisely come into contact with actual world, would be turned into a mere heuristic device for structural knowledge.

\section{Bringing in patterns while striving for realism}

Eddington conceived of physical reality as a "synthesis of appearances" integrating into one single scheme as many aspects as possible of the world, inasmuch as the "relativistic outlook" aims at achieving the "point of view of no one in particular" 47 . This process of gradual enrichment of the world image has been depicted by Eddington himself as analogous to the sequence leading from monocular to binocular static vision, and then to the enhanced view performed when different perspectives mesh ${ }^{48}$. As one may currently think of varying position, motion and magnitude-scale of the observer, more and more comprehending geometric frameworks progressively emerge. Minkowski space-time corresponds then to the world as "seen" by observers who may be granted all possible uniform velocities, whereas owing to Riemannian geometry, Einstein's general relativity encapsulates the whole range of observers' viewpoints whatever be their velocities, while, eventually, Weyl's gauge principle would complete the whole relativity scheme by allowing shrinking or expanding observers. Tensors were then meant to be the appropriate tool for achieving such a synthesis, since they provide, as [35, p. 190] elegantly puts it, "a means of winnowing the wheat of 'intrinsic information' about the absolute four-dimensional world from the extrinsic chaff of particular perspectives". Since what makes a tensor is nothing else but the lawlike way in which a set of quantities relative to a given coordinate system transforms itself into another set of corresponding quantities, when one shifts to a different coordinate system, tensor equations are invariant under coordinate transformations. Though a tensor as such does not afford any measure of the intrinsic properties of the physical world, except of course in the case of scalar tensors, linear equations involving tensors do grasp these. Insistence on such an epistemic feature of tensors conceived as giving access to the core of physical reality may have been actually more or less entrenched in mathematical practice and thus rather current at the time ${ }^{49}$, since recourse to tensors had been spurred precisely by the urge to decide how physical statements may express a content which would be independent of any arbitrarily chosen coordinate system. But the fact that this concern about physical reality eventually resulted in giving structure priority over substance is certainly peculiar to Eddington's

\footnotetext{
$46[8$, p. 200].

$47[35, \S 7.3]$

${ }^{48}$ Cf. [8, p. $\left.182-183\right]$

${ }^{49} \mathrm{Cf}$. in particular $[45, \S 5]$.
} 
view in the early twenties. Relativity theory would indeed allegedly be held to require a "higher order synthesis" yielding an 'absolute' world which, while "not [to be thought of as] mind independent for it is composed of the 'synthetic unity' of all its relative aspects", would still be granted a form of unity presumably "not representable in space and time, but only as a structure in a geometrical conceptual space" 50 .

\subsection{Chasing the pucker}

Inasmuch as he originally favoured a realist interpretation of the invariants of general relativity theory by endowing them with an absolute significance, the main thrust of Eddington's argument consisted in denying any ultimate justification to the distinction between allegedly 'real' and 'unreal' fields of force, thus looking for deeper reality as embodied in structural space-time features fixed by differential equations. As Eddington stressed in his lively exposition of relativity theory for the layman, it is for instance customary to oppose gravitational and centrifugal forces by claiming that the first are inescapable whereas the second presumably come from curvature of track. But since the criterion allowing to deem artificial a certain field of force boils down to its possible annulling through choosing an appropriate observer, demarcating gravitation from centrifugal force is in fact to be deprived of all grounds in Eddington's view. It would indeed be as much possible to remove gravitation - though piling it up somewhere else - than centrifugal force, the only remaining difference being that the first is removable only locally whereas the second can be removed everywhere. Since "gravitation is equally unreal with centrifugal force" ${ }^{51}$, reality must then be sought at a deeper level. Not only does Eddington underscore as "a remarkable fact that in a limited region, it is possible to create an artificial field of force which imitates a natural gravitational force so exactly that, so far as experiments have yet gone, no one can tell the difference" ${ }^{2}$, but he also gives the "principle of equivalence" an unequivocal formulation by stating that "a gravitational field of force is precisely equivalent to an artificial field of force, so that in any small region it is impossible by any conceivable experiment to distinguish them" ${ }^{53}$. Though likewise formulations have since been claimed unintelligible ${ }^{54}$ or even false since, as shown by [29], gravitational field may arguably be detected through its tidal effects ${ }^{55}$, Eddington seems to have endorsed them as a link in his reasoning. A gravitational field

\footnotetext{
50 [35, p. 187].

51 [8, p. 67], my emphasis.

$52[8$, p. 64].

53 [8, p. 76].

${ }^{54} \mathrm{Cf}$. [40, p. IX] : "I have never been able to understand this Principle ... Does it mean that the effects of gravitational field are indistinguishable from the effects of an observer's acceleration ? If so, it is false. In Einstein's theory, either there is a gravitational field or there is none, according as the Riemann tensor does not or does vanish. This is an absolute property; it has nothing to do with any observer's world-line ... The Principle of Equivalence performed the essential office of midwife at the birth of general relativity, but, as Einstein remarked, the infant would never have gone beyond its long clothes had it not been for Minkowski's concept [of space-time geometry]. I suggest that the midwife be now buried with appropriate honours and the facts of absolute space-time faced".

${ }^{55} \mathrm{I}$ am grateful to an anonymous referee for pointing out to me the relevant paper by $\mathrm{H}$. C. Ohanian, [29, p. 903-904] : "it is generally believed that the effects of curvature will only manifest themselves in regions of space-time of sufficiently large size. Our intuition suggests that as long as we explore only a very small region, the curved space-time can be approximated with arbitrary precision by a flat spacetime. In Newtonian gravitation, this amounts to neglecting tidal effects. But in fact, our intuition lets us down very badly. (...) we will present several simple examples which show that in some systems
} 
being thus held practically indistinguishable from an artificial field of force in any sufficiently small neighbourhood, the geometry of the four-dimensional world can equivalently be explored either by practically tracing geodesics or by measuring space-time intervals by means of unconstrained measuring appliances, viz. free-falling clocks and rods. Therefore, since it can be flattened out by coordinate change, the gravitational field thought of as the measured field has no absolute character whatsoever, but curvature does. The ten potentials of the metric tensor $d s^{2}=g_{\mu \nu} d x^{\mu} d x^{\nu}$ do encapsulate the actual spacetime geometry in their own specific involved way which Eddington described vividly by picturing the space-time in the gravitational field round matter as a pucker which can be pressed out flat and shifted, but never suppressed since it always runs somewhere else. "What determines the existence of the pucker is not the values of the $g$ 's at any point, or, what comes to the same thing, the field of force there. It is the way these values link on to those at other points - the gradient of the $g$ 's and more particularly the gradient of the gradient" ${ }^{\prime 6}$. At this stage, curvature, action and matter form a network of closely interrelated concepts, for not only "action is the curvature of the world" but also "wherever there is matter there is action and therefore curvature" 57 , since curvature can be represented by the Einstein tensor which in return is to be equated with matter.

\subsection{Matter is provisional hodge-podge}

As is well known, analogy with Poisson's equation served as a guiding principle to frame out the gravitational field equations. Einstein took it explicitly as a model of what had to be done in relativity theory. Poisson's equation $\nabla^{2} \phi=4 \pi G \rho$ can be seen as the Newtonian law of gravitation couched in terms of potential theory, and it expresses the idea that the gravitational field arises from the density $\rho$ of ponderable matter. But then a new source term corresponding to stress-energy density, viz. the energy tensor $T_{\mu \nu}$, has to be substituted in the right hand side of the equation for the scalar density of matter $\rho$, whereas the left hand side tensor which is to be built out of the $g_{\mu \nu}$ must be constrained by three conditions stemming from the analogy with Poisson's equation ${ }^{58}$, namely that 1 . it is not allowed to contain differential coefficients of the $g_{\mu \nu}$ higher than the second, 2. it has to be linear in them, and 3. its divergence must vanish identically. Tensors satisfying the first two conditions are proved to conform to one single pattern $R_{\mu \nu}-a . g_{\mu \nu} R$, where $R_{\mu \nu}$ denotes the Ricci curvature tensor obtained by contraction from the Riemann tensor and $R$ is the scalar curvature $R=g^{\mu \nu} R_{\mu \nu}$, whereas the third condition imposes the parameter's value $-\frac{1}{2}$. The whole deductive process thus ends up with Einstein's field equations

$$
R_{\mu \nu}-\frac{1}{2} g_{\mu \nu} R=-\kappa T_{\mu \nu}
$$

which in return admit of two different interpretations, depending on whether one reads them from right to left or from left to right. The first way suggests that energy determines

\footnotetext{
the effects of curvature never do disappear, not even in the limiting cases of systems of infinitesimal size. All these examples involve very well-known physical effects which can be discussed entirely in the framework of Newtonian gravitational theory, however the relevance of these effects to the question of equivalence of inertia and gravitation has not been sufficiently appreciated."

$56[8$, p. 85$]$.

$57[8$, p. 148].

${ }^{58}$ Cf. [17, p. 48$]$
} 
or shapes geometry, while the second embodies the reverse view according to which the fixing of the $g_{\mu \nu}$ defines eo ipso the energy content of the world. Comparing his own equations to "a building one wing of which is made of fine marble (left hand part of the equation), but the other wing of which is built of low grade wood (right hand side of the equation)" 59 , Einstein himself thought of the phenomenological representation of matter, viz. $T_{\mu \nu}$, as a pis aller, since it was "only a crude substitute for a representation which would correspond to all known properties of matter" ${ }^{60}$. He thus emphasized that the right-hand side of the field equations was nothing but an unsatisfactory aggregate intending to cement different things whose ultimate nature was still unknown.

We have seen, indeed, that in a more complete analysis the energy tensor can be regarded only as a provisional means of representing matter. In reality, matter consists of electrically charged particles (...) It is only the circumstance that we have no sufficient knowledge of the electromagnetic field of concentrated charges that compels us, provisionally, to leave undetermined, in presenting the theory, the true form of this tensor. From this point of view our problem now is to introduce a tensor, $T_{\mu \nu}$, of the second rank, whose structure we do not know but provisionally, and which includes in itself the energy density of the electromagnetic field and of ponderable matter. ${ }^{61}$

Einstein would later be even more explicit in his Autobiographical Notes, when he insists that the right hand side of the field equation was "a formal condensation of all things whose comprehension in the sense of a field theory is still problematic", the whole formulation being "a makeshift in order to give the general principle of relativity a preliminary closed-form expression", for it was essentially no more than "a theory of the gravitational field, which was isolated somewhat artificially from a total field of as yet unknown structure" 62 . These cautious remarks by Einstein suggest in particular that a more fine-grained analysis of the true form of this tensor would allegedly require a better understanding of how general relativity and quantum mechanics might mesh.

\subsection{Analogizing permanence}

However, Eddington seems to have deliberately overlooked such intricacies, at least at a first stage in the early twenties, since he squarely interpreted field equations as providing a definition of matter while justifying his use of such a principle of identification by bringing to the fore the process of analogizing permanence. He thus deemed the vanishing of the divergence of the Einstein tensor ${ }^{63}$, better known to physicists as the contracted Bianchi identities, the "fundamental theorem of mechanics" inasmuch as it allowed such an identification step.

The divergence of $G_{\mu}^{\nu}-\frac{1}{2} g_{\mu}^{\nu} G$ is identically zero. In three dimensions the vanishing of the divergence is the condition of continuity of flux, e. g. in hydrodynamics 
$\partial u / \partial x+\partial v / \partial y+\partial w / \partial z=0$. Adding a time-coordinate, this becomes the condition of conservation or permanence... It will be realised how important for a theory of the material world is the discovery of a world-tensor which is inherently permanent. ${ }^{64}$

Mathematics thus provides a well-behaved tensor that summarizes the common properties of matter pertaining to mass, momentum and stress, and it is indeed all that is needed for Eddington's world-building purposes. While discarding matter as an independent substance, Eddington thence claims that ci-devant phenomenological matter is to be taken in charge by would-be substantial tensors.

Moreover it is not an accident that it should be this particular tensor [i.e. $\left.G_{\mu}^{\nu}-\frac{1}{2} g_{\mu}^{\nu} G\right]$ which is capable of being recognised by us. It is because its divergence vanishes - because it satisfies the law of conservation - that it fulfils the primary condition for being recognised as substantial. If we are to surround ourselves with a perceptual world at all, we must recognise as substance that which has some element of permanence. We may not be able to explain how the mind recognises as substantial the world-tensor $G_{\mu}^{\nu}-\frac{1}{2} g_{\mu}^{\nu} G$, but we can see that it could not well recognise anything simpler. There are no doubt minds which have not this predisposition to regard as substantial the things which are permanent; but we shut them up in lunatic asylums. ${ }^{65}$

Pattern recognition is thus the mind's own contribution though a "real objective substratum" is given, as the eye only picks but a few selected patterns out of the unbounded variety of those which could be traced in the starry heavens and in a certain way are there independently of our grasping them. $G_{\mu}^{\nu}-\frac{1}{2} g_{\mu}^{\nu} G$ is one of these and, as Eddington lively puts it, "where it can trace it, the mind says : 'Here is substance'; where it cannot, it says 'How uninteresting ! There is nothing in my line here'." 66 The seed of selective subjectivism thus seems to have been already sown at this stage in the soil of Eddington's rooted realism. Later on, in the thirties, Eddington would develop such a view as an independent full-fledged doctrine by gradually emancipating it from these early twenties bonds of faithfulness to original concerns for physical reality. Among many others, a striking illustration of this mature stance was given in the form of a parable in which Eddington imagines an ichthyologist who would explore the depths of the ocean and, by inspection of what would come up in his fish nets, would formulate two general laws out of his catch : 1. "no-creature is less than two inches long"; and 2. "all sea-creatures have gills". To whom may contest the first claim, the ichthyologist would answer : "what can't be caught in my nets is beyond ichthyological knowledge", and so does Eddington aver analogously that "generalisations that can be reached epistemologically have a security which is denied to those that can only be reached empirically" 67 . Inasmuch as he eventually embraced this view of the mind selecting a priori patterns as objective features amidst empirical reality, Eddington verged to sidestep how mathematical theory comes into contact with the actual world, though he originally addressed this issue by the early twenties in his case for "Hamiltonian derivatives".

\footnotetext{
${ }^{64}[12, \S 52$, p. 115$]$, the emphasis is Eddington's.

$65[12, \S 54$, p. 120], my emphasis.

$66[10$, p. 420$]$

$67[14$, p. 19$]$
} 


\section{3. "Hamiltonian derivatives" and their dual role}

\subsection{Making the mind collude with nature}

According to Eddington's view, as recapitulated at a first stage in [12, 8 64], mathematical theory can be tied up with the physical world either (1) by identifying the mathematical interval with a quantity which is the result of practical measurement with scales and clocks, or (2) by identifying the tensor $G_{\mu}^{\nu}-\frac{1}{2} g_{\mu}^{\nu} G$ with phenomenological matter, namely "with the mechanical abstraction of matter which comprises the measurable properties of mass, momentum and stress" 68 . These two points of contact then make it possible to build a "complete cycle of reasoning" 69 by pasting together two different trains of thought : a purely deductive chain which consists in deriving the energy tensor from the space-time interval and a reverse chain passing from matter as defined by the energy tensor to the interval thought of as resulting from measurements made with appliances built out of matter thus conceived. The full completion of this second chain would then bring about something more than what is encapsulated in the energy tensor, since measurement requires the existence of discrete particles ${ }^{70}$. However atomicity of matter comes into the picture as crucially connected with the issue of realism. Reading the field equations from left to right and hence identifying matter with the energy tensor would have two main consequences, as claimed by [20, p. 230], namely (1) eliminating substance from our ontology in favour of relational structures, and (2) emphasizing the dependence on the mind, of matter as identified with the energy tensor. But assuming that pattern recognition is really contributed by the mind, why should mathematical identities constrain the external world whatsoever ? Granting atomicity would indeed entail acknowledging the fact that there are laws in the external world, since "we have not learnt why the quality called matter exists only in certain lumps, called atoms or electrons" ${ }^{71}$. Hence, if mind cannot account for atomicity, what could account for it ?

A more likely suggestion is that our analysis into point events is not final; and if we would carry the analysis beyond the point event to something still more fundamental, then atomicity and the remaining laws of physics would become obvious identities. This may well be the case. ... But if we attempt to push the analysis behind the point events, we are, I think, bound to particularize the structure. ${ }^{72}$

Along these lines, [20] claims that, though atomicity seems to reflect non structural substance, "the structural account of atomicity [would] remove even this substantial element" 73 , resulting later in Eddington's mature structuralism owing to a better understanding of how general relativity and quantum theory engage with one another. Whereas obviously hinting at such a structuralist account of atomicity, Eddington nevertheless suggests following a different track in the early twenties.

Matter is a property of the world to which the human mind attributes an exaggerated importance for reasons which Nature would regard irrelevant; yet she seems

\footnotetext{
$68[12, \S 64$, p. 146].

69 [12, p. 146]

${ }^{70} \mathrm{Cf}$. [35, p. 208] for details.

$71[9$, p. 156$]$

$72[9$, p. 157].

$73[20$, p. 232$]$
} 
to be in collusion with mind in singling out this property for atomicity. I can only suggest that the difficulty might disappear if we understood better the true relation between atomicity of matter and the more general atomicity which underlies all quantum phenomena. As far as we can understand it at present, there is some kind of atomicity of the quantity known in mechanics as Action, and this seems to be the fundamental origin of all atomic phenomena. If so, that must be Nature's own idea, for which she is in no way indebted to us. (...) If then we can account for the apparent atomicity of matter as resulting from the quanta of Action, the difficulty alluded to will disappear; but this is at present a speculation. ${ }^{74}$

$[12, \S 60]$ made this speculation more precise owing to what Eddington coined "Hamiltonian derivatives" which were intended to embody the mind's collusion with nature by linking matter with action. Such variational derivatives are thus not to be thought of solely as "expressing Mind's regard for permanence" 75 or as providing the means to make the theoretical and the mental world-building converge ${ }^{76}$, but also as tuning up mind's privileged pattern to "Nature's own idea", as far as Eddington meant the role ascribed to "Hamiltonian differentiation" to be dual.

\subsection{The noble art of "Hamiltonian differentiation"}

Action is usually defined in mechanics as energy multiplied by time. But from a broader point of view, as Eddington laid much stress on in [12, §60, p. 138], action can be envisaged under two different aspects, namely (1) as a physical quantity which has a definite numerical value and is of special significance since it is invariant, or (2) as a mathematical function of some variables, whose functional form depends on the variables in terms of which the action is to be expressed. Starting with the invariant integral representing gravitational action in a four-dimensional space-time region

$$
\mathbf{A}=\iiint \int G \sqrt{-g} d \tau
$$

where $g=\operatorname{det} g_{\mu \nu}$, and $G=g^{\mu \nu} G_{\mu \nu}$, described as the "Gaussian curvature" 77 , is the Ricci scalar, Eddington considers the variation of the integral, $\delta \mathbf{A}$, for arbitrary small variations of the variables, $\delta g_{\mu \nu}$, which vanish at and near the boundary of the given region and thus obtains the following relation :

$$
\delta \int G \sqrt{-g} d \tau=-\int\left(G^{\mu \nu}-\frac{1}{2} g^{\mu \nu} G\right) \delta g_{\mu \nu} \sqrt{-g} d \tau .
$$

The coefficient $-\left(G^{\mu \nu}-\frac{1}{2} g^{\mu \nu} G\right)$, now known as the variational derivative, is what Eddington calls the "Hamiltonian derivative" of $G$ with respect to $g_{\mu \nu}$, which he denotes as follows

$$
\frac{\mathbf{h} G}{\mathbf{h} g_{\mu \nu}}=-\left(G^{\mu \nu}-\frac{1}{2} g^{\mu \nu} G\right)=8 \pi T^{\mu \nu} .
$$

\footnotetext{
${ }^{74}[9$, p. 158], my emphasis.

$75[35, \S 7.5 .2]$

$76[20$, p. 234$]$

${ }^{77}$ Recall that Eddington denotes the Ricci tensor and the scalar curvature tensor with $G$ 's instead of $R$ 's as is usual nowadays.
} 
Though relying on ideas which, as seen above, can be traced back to Hilbert who first used variational methods to derive gravitational field equations, Eddington nevertheless moulded his own interpretation of Hamilton's variational principle by bringing to the fore a key property of variational derivatives. One can indeed apply just the same method to any invariant $K$ and yield a variational derivative of $K$ with respect to $g_{\mu \nu}$, namely

$$
\frac{\mathbf{h} K}{\mathbf{h} g_{\mu \nu}}=P^{\mu \nu}
$$

Eddington then proves that the variational derivative of any fundamental invariant is a tensor whose divergence vanishes ${ }^{78}$, which thus makes variational derivatives in general the appropriate means for conveying permanence and embodying the mind's privileged pattern. But at the same time, this essential property opens the way to alternative energy tensors, since one could have started equivalently with these three fundamental invariants $^{79}$ :

$$
K=G, \quad K^{\prime}=G_{\mu \nu} G^{\mu \nu}, \quad K^{\prime \prime}=B_{\mu \nu \sigma}^{\rho} B_{\rho}^{\mu \nu \sigma},
$$

thus giving rise to "three alternative material worlds" 80 between which it is impossible to decide on observational grounds, since observation boils down to schedules of pointerreadings and "after all it is the relation which is the reality" 81 . As far as systems of coincidences are the only available evidence, all these alternative tensors allegedly do the same thing and are indeed equivalent, so that the criterion of simplicity seems to be the only guiding principle left to make a choice and pick out the Ricci scalar.

Since the early twenties, modifications of Einstein's theory of gravity induced by such alternative Lagrangians leading to higher-order field equations have received much attention over years, though with alternating high and low tides depending on the way the pros and cons of the corresponding prospects were assessed. With hindsight, four different steps may advantageously be distinguished insofar as subsequent developments presumably unfold some of the implicit grounds supporting Eddington's claim that such possibly alternative theories of gravity cannot be observationally demarcated.

(1) Shortly after the advent of general relativity, while opening the way to the exploration of such alternative general relativistic theories of gravitation, Weyl ([44], [43, sec. $35 \& 36]$ ) underscored the general significance of quadratic Lagrangians for a possible extension of relativity theory and, in so doing, not only suggested the two invariants which Eddington takes into account in addition to the Ricci scalar, namely in current notation $L_{2}=R_{\mu \nu} R^{\mu \nu}$ and $L_{3}=R_{\mu \nu \rho \sigma} R^{\mu \nu \rho \sigma}$, but also the one obtained directly from the Ricci scalar, viz. $L_{1}=R^{2}$. Bach ([1]) then focussed on such invariants and their combinations and showed how they can be reduced to a small number of them. Anyway Weyl would later prove that all such alternative Lagrangians give rise to fourth order vacuum field equations by contrast with the standard Einstein second order ones based on the Ricci scalar $^{82}$, which was presumably part of the rationale for choosing the Ricci scalar as the base invariant $K$ in the action principle (cf. equ. (7)). Still, other choices for $K$ would

\footnotetext{
${ }^{78}$ Cf. $[12, \S 61$, p. 140$]$

${ }^{79}$ As will be seen below, Eddington denotes the Riemann-Christoffel tensor by $B_{\mu \nu \sigma}^{\rho}$.

$80[12, \S 62$, p. 141$]$.

81 [12, p. 144]

${ }^{82}$ Cf. [43, Appendix II].
} 
also imply "admissible laws of gravitation" 83 since these alternative Lagrangians would accordingly lead to contracted Bianchi identities and would thus satisfy the fundamental theorem of mechanics. How then would it be possible to discriminate between such alternative laws if "any field of gravitation agreeing with Einstein's law would also satisfy the alternative law proposed, [though] not usually vice versa" ${ }^{84}$ ? Eddington thus established for instance that the Schwartzschild's solution is also a solution of the alternative equations

$$
\frac{\mathbf{h} L_{2}}{\mathbf{h} g_{\mu \nu}}=0, \quad \frac{\mathbf{h} L_{3}}{\mathbf{h} g_{\mu \nu}}=0,
$$

and later Buchdahl ([4, p. 90]) would also prove the same for

$$
\frac{\mathbf{h} L_{1}}{\mathbf{h} g_{\mu \nu}}=0 .
$$

(2) More generally, Pauli ([30]) had already shown that every vacuum solution, including the Schwarzschild one, of Einstein's field equations is also a solution of

$$
\frac{\mathbf{h} L_{1}}{\mathbf{h} g_{\mu \nu}}=0, \quad \frac{\mathbf{h} L_{2}}{\mathbf{h} g_{\mu \nu}}=0,
$$

Since, as noted by [22, p. 634], the Schwarzschild solution of Einstein's equations is the basis of the classical tests of general relativity ([31]), Pauli could argue that the equations lead to no observational disagreement with Einstein's theory regarding the shift of Mercury's perihelion and the light deviation in the solar gravitational field ${ }^{85}$. Presumably, this is the basis of Eddington's claim that the alternative theories cannot be distinguished by any "schedules of pointer-readings".

(3) Still, only later results would shed some light on the hidden rationale behind Eddington's insight :

When we ask which of the three [admissible laws of gravitation] is the law of the actual world, I am not sure that the question has any meaning ... One cannot but suspect that there is some identical relation between the Hamiltonian derivatives of the three fundamental invariants. If this relation were discovered it would perhaps clear up a rather mysterious subject. ${ }^{86}$

From the thirties on, Lanczos advocated quadratic Lagrangians as a basis for unified field theory inasmuch as "field equations of nature" would presumably be deducible from a scale invariant action principle ([26]) and, in support of his view, he had indeed proved already in the late thirties ([25]), that the general possibilities for such alternative Lagrangians reduce to the two only effective invariants $L_{1}=R^{2}$ and $L_{2}=R_{\mu \nu} R^{\mu \nu}$ and combinations thereof, since all the others (in particular $L_{3}=R_{\mu \nu \rho \sigma} R^{\mu \nu \rho \sigma}$ ) prove inactive in the formation of field equations. More precisely, when studying the action principle $\delta \int I d \tau=0$ which controls the field equations, Lanczos assumes that the fundamental

\footnotetext{
${ }^{83}[12$, p. 143$]$

$84[12$, p. 143$]$.

${ }^{85}$ Cf. [41, p. $\left.105-106\right]$.

86 [12, p. 144].
} 
invariant $I$ is to be a merely algebraic function of the components of the RiemannChristoffel tensor $R_{\mu \nu \rho \sigma}$ and, owing to combinatorial considerations, proves first that there are five apparently independent invariants suitable for action principle, namely the usual $L_{1}, L_{2}, L_{3}$ plus two additional ones $K_{1}$ and $K_{2}$. He then shows that "this surplus of invariants is only apparent" inasmuch as "the invariants $K_{1}$ and $K_{2}$ do not contribute any terms to the field equations since their variation reduces to a mere boundary term" 87 and thus obtains in particular the following linear relation between the invariants $L_{1}$, $L_{2}, L_{3}$ and $K_{2}$ :

$$
K_{2}=L_{3}-4 L_{2}+L_{1}
$$

Since the field equations deduced from $K_{2}$ by action principle vanish identically, the field equations implied by the variation of the action integral $\int L_{3} d \tau$ are identical with those derived from the action integral $\int\left(4 L_{2}-L_{1}\right) d \tau$. As the true source from which stem the identities alluded to, the linear relation between the fundamental types of alternative invariants $(e q u .(15))$ then encapsulates the reason why the fourth-order equations based on $L_{1}, L_{2}, L_{3}$ contain the solutions of Einstein's second-order equations and thus, as noted by [22, p. 634], justifies "Eddington's mathematical hunch".

(4) However, the viability of such alternative gravitational theories came to be eventually disclaimed with respect to their weak-field behaviour in the sixties and the seventies. Buchdahl ([5]) grounded his rebuttal of gravitational theories derived from a pure $R^{2}$ quadratic Lagrangian on the fact that the corresponding field equations cannot represent space asymptotically flat at infinity. Though, as explained above, the Schwarzschild's solution allowed one, in the case of vacuum field equations, to argue in the sense of observational equivalence of alternative gravitational theories inasmuch as providing a common base for experimental tests, Bicknell ([2]) showed that, when one brings in a stress-energy tensor, predictions differ markedly from observations, not only for theories based on pure quadratic Lagrangians $R^{2}$, but also for those derived from the most general Lagrangian, viz. $R_{i j} R^{i j}-\nu R^{2}$ (owing to Lanczos result above), since weak-field approximation makes it clear that in such Lagrangians the $R^{2}$ term dominates. From a different viewpoint, Havas ([22]) established that in the presence of extended sources, higher-order alternative theories contradict Newton's law of gravitation in the non relativistic limit, unless one allows for asymptotical agreement with additional terms, and even in this case, such generalizations of Einstein's law would not yield static spherically symmetric solutions of Schwarzschild's form thus failing to comply with Birkhoff's theo$\mathrm{rem}^{88}$. Since the main problem concerns extended mass distribution, Havas significantly comments on Eddington's acknowledging the fact that fourth-order vacuum equations admit more than one type of spherically symmetric solutions, though failing to recognize that only one of these is the limit of the solution for an extended source. After having put forward the Schwarzschild's solution, Eddington remarked that "there are doubtless other symmetrical solutions for the alternative laws of gravitation which are not permitted by Einstein's law, since the differential equations are now of the fourth order and

\footnotetext{
87 [25, p. 845].

${ }^{88}$ I thank an anonymous referee for drawing my attention to this point and for mentioning the relevant paper by Havas. In this connection, one may recall, as explained in [22, p. 640], that, for instance in the case of a single spherically symmetric body in Einstein's theory, Birkhoff's theorem states that "the field exterior to the body is independent of its size and internal stresses and is identical to the static spherically vacuum solution".
} 
involve two extra boundary conditions either at the particle or at infinity" ${ }^{\prime 89}$, without noticing though that such other symmetrical solutions should be related to the solutions with extended sources ${ }^{90}$. On the base of such results, one might thus be inclined to take Eddington's claim of the observational indistinguishability of alternative gravitational theories cum grano salis ${ }^{91}$.

\subsection{How "Hamiltonian derivatives" do work out what they are meant for}

Whatever be the case, there is nevertheless another feature of variational derivatives which should be neither downplayed nor misinterpreted, for it plays the essential role in allowing the mind's collusion with nature. Integrals for which variational derivatives vanish have a constant value. Therefore, in the remarkable case of action, one might be tempted to acknowledge this fact as a stronghold in favour of the principle of stationary action, but one would then simply miss the point, since, in Eddington's view, "just as in the ordinary differential calculus, we are not solely concerned with problems of maxima and minima, and we take some interest in differential coefficients which do not vanish; so Hamiltonian derivatives may be worthy of attention even when they disappoint us by failing to vanish"92. But what happens when variational derivatives do not vanish? And how their virtual non-vanishing may help linking atomicity of matter with atomicity of action? At first sight, the principle of stationary action seems to be at odds with the derivation of Einstein's field equations through variational differentiation, since action cannot be stationary but in empty space, namely when $T^{\mu \nu}$ vanishes.

It would thus appear that the Principle of Stationary Action is in general untrue. Nevertheless some modified statement of the principle appears to have considerable significance. In the actual world the space occupied by matter (electrons) is extremely small compared with the empty regions. Thus the Principle of Stationary Action, although not universally true, expresses a very general tendency - a tendency with exceptions. Our theory does not account for this atomicity of matter;

\footnotetext{
89 [12, p. 143].

${ }^{90}$ Havas luminously points out that this problem is "closely related to the one which caused Newton to delay publication of the Principia for two decades" ([22, p. 639]), namely the issue concerning the relation between a postulated force law between two mass points and the corresponding force law between two homogeneous spheres. "This problem is independent of any field equations satisfied by the force or the corresponding potential; however, if such equations, rather than elementary force laws, are taken as basic, a new question arises, namely, whether these equations admit more than one type of spherically symmetric solutions. In the case of the Laplace's equation only one such type of solution exists, and the resultant $1 / r$ potential between two point masses $M_{1}$ and $M_{2}$ is identical to the potential between two homogeneous spheres with centers at a distance $r$ apart and of masses $M_{1}$ and $M_{2}$ and radii $R_{1}$ and $R_{2}$ respectively. While this Newtonian result appears trivial even from the vantage point of 19 th century mathematics, it was noted only recently that there is no other potential with these properties. No potential between point particles other than one proportional to $e^{-\lambda r} / r$ yields a potential between spheres that has the same dependence on $r$, and only if $\lambda$ vanishes (the Newtonian case) is the coefficient of the resultant potential independent of the radii" ([22, p. 640]). The thrust of the argument is then to argue that there is no such theorem in the case of higher-order field equations arising from alternative Lagrangians.

${ }^{91}$ Nevertheless, to conclude this point with a cautious remark, this verdict of non-viability may perhaps not be ultimate inasmuch as challenged to a certain extent by recent developments ([6]) which revive interest in such gravity theories with quadratic Lagrangians by working out the Newtonian limit of fourth-order gravity along a metric approach requiring a different framework.

92 [12, p. 138]
} 
and in the stationary variation of action, we seem to have an indication of the way of approaching this difficult problem, although the precise formulation of the law of atomicity is not yet achieved. It is suspected that it may involve an "action" which is capable only of discontinuous variation. ${ }^{93}$

The non-vanishing of the variational derivatives thus pinpoints the possible juncture of general relativity and quantum theory. Variational derivatives do indeed vanish on the average though they may fail to vanish at some definite spots, thus making atomicity of matter compatible with stationary action by granting that both properties do not conflict since they stand at two different levels. The scheme of things thus provided is to be thought of as a provisional overall framework which does not preclude nor anticipate further refinements, since, as Eddington warns, "the possibility of the existence of an electron in space is a remarkable phenomenon which we do not yet understand", and "the details of [whose] structure must be determined by unknown equations" 94 .

Technically, variational derivatives combine two different kinds of features, namely (1) they may not vanish at the fine-grained level while vanishing on the average, and at the same time (2) their divergence always vanish. This peculiar conjunction of properties is precisely what suit them for the dual role of making mind collude with Nature, since the hunger for permanence which characterizes the mind can then be hooked to the "creative aspect" of these "Hamiltonian derivatives" which reveals nature's contribution. Though the law of conservation results from the mind's demand, Eddington stresses the fact that "we [not only] might have built things which do not satisfy this law" but "in fact we do build one very important thing, 'action', which is not permanent", so that "in respect to "action", physics has taken the bit in her teeth, and has insisted on recognising this as the most fundamental thing at all, although the mind has not thought it worthy of a place in the familiar world and has not vivified it by any mental image or conception" 95 .

But since the non-vanishing of the infinitesimal variation of gravitational action $\delta \mathbf{A}$ (cf. equ. (7)) entails the non-vanishing of the variational derivative and hence of the energy tensor $T^{\mu \nu}$ (cf. equ.(8)), variational derivatives do link atomicity of action with atomicity of matter. Besides, $\delta \mathbf{A}$ being the variation of the action integral over a four dimensional space-time region, its non vanishing can be seen as a sparking event within the boundaries of this region, which grounds Eddington's claim that "Hamiltonian derivatives" are to be endowed with a "creative character".

It is a feature of our attitude towards nature that we pay great regard to that which is permanent; and for the same reason the creation of anything in the midst of a region is signalised by us as more worthy of remark than its entry in the orthodox manner through the boundary. Thus when we consider how an invariant depends on the variables used to describe the world, we attach more importance to changes which result in creation than to changes which merely involve transfer from elsewhere. It is perhaps for this reason that the Hamiltonian derivative of an invariant gives a quantity of greater significance for us than, for example, the ordinary derivative. The Hamiltonian derivative has a creative quality, and thus stands out in our minds as an active agent working in the passive field of space-time. Unless this idiosyncrasy of our practical outlook is understood, the Hamiltonian method

\footnotetext{
93 [12, p. 139]

$94[12$, p. 153$]$

$95[13$, p. 241]
} 
with its casting away of boundary integrals appears somewhat artificial; but it is actually the natural method of deriving physical quantities prominent in our survey of the world, because it is guided by those principles which have determined their prominence. ${ }^{96}$

Up to this stage, variational derivatives were meant to implement a speculative unificatory scheme by homogenizing sparkles of discontinuous action into uniform field equations, owing to inherent mathematical features that happen to be adequate for this purpose, and by the same token, they were supposed to grant mind's adherence to external reality.

As he tried to accommodate his view to Weyl's ideas regarding pure infinitesimal geometry and gauge invariance, Eddington however shifted, at a further stage of his developing relativity theory, from a world-building which would proceed along Einstein's lines from the fundamental metric tensor $g_{\mu \nu}$, to an allegedly non metrical world geometry which would be entirely derived from the mere assumption of a metric independent affine connection. Within this new framework, the method of identification would deal with gauge invariant tensors and customary action would be discarded as an invariant, therefore variational derivatives would go out of tune and fit no more mind's pattern to nature's proper initiatives.

\section{Going out of tune through gauge invariance}

Though he credited Weyl for having contributed to complete the relativistic outlook by removing the last remnant of Euclidean "distant geometry" that still prevented Riemannian geometry from being a pure infinitesimal geometry, Eddington adopted an original view which "entirely alters the status of Weyl's theory", since it presents it as "no longer a hypothesis, but a [mere] graphical representation of the facts" 97 whose value lies in the insight it suggests.

\subsection{Gauge transformations}

While denying the tacitly assumed comparability of lengths at different points, Weyl brought in the gauge concept in order to account for non-integrability of length by generalizing Riemannian metric. Since the length of a vector may change depending on the path which is followed when it is transferred from a point $P$ to a nearby point $P^{\prime}$, Weyl's method consists in fixing a unit of length, viz. a "gauge", at each point of the space-time manifold, so that the variation of length may be measured by comparing the length with the gauges attached to the successive points at which the vector is to be located along its path. Since gauge systems may be arbitrarily chosen just like coordinate systems, gauge change means multiplying lengths by a scale coefficient, $\lambda(x)$, which is a strictly positive real valued function ranging over space-time manifold points, viz.

$$
\bar{l}=\lambda(x) . l \quad \text { and } \quad \bar{g}_{\mu \nu}=\lambda(x)^{2} g_{\mu \nu}
$$

By analogy with the affine connection which fixes the meaning of Levi-Civita parallel displacement owing to the vanishing of the covariant derivative, Weyl then defines the

\footnotetext{
96 [12, p. 147], my emphasis

$97[12$, p. 208]
} 
length connection as allowing congruent displacement ${ }^{98}$. Assuming that a vector $A^{\mu}$ is transferred from a point $P$ to a nearby point $P^{\prime}$, and comparing its original length $l$ measured by the gauge at $P$ with its ultimate length $l+\delta l$ measured by the gauge at $P^{\prime}$, the change of length, $\delta l$, can be partly ascribed to the shifting of position on the one hand and to the recalibrating of the unit length imposed by the gauge system on the other hand, which, as [35, p. 152] explains, corresponds to the following stipulation

$$
\delta l:=\frac{\partial l}{\partial x^{\mu}} \cdot d x^{\mu}+l . d \phi
$$

Congruent displacement then suggests the vanishing of the $\delta l$ variation, so that $d l=$ $-l d \phi$ holds, provided that the two parts, namely the shifting of the position and the recalibrating of the unit of length, may be supposed independent, and that the first part $\frac{\partial l}{\partial x^{\mu}} \cdot d x^{\mu}$ may be replaced by the complete differential $d l$. The same relation holds, when the length is recalibrated by choosing an alternative gauge system, $\bar{l}=\lambda . l$, since $d \bar{l}=-\bar{l} . d \bar{\phi}$ stems obviously from the Leibniz rule $d \bar{l}=d \lambda . l+\lambda . d l$, so that the multiplying factor depending on the new gauge system is such that $d \bar{\phi}=d \phi-\frac{d \lambda}{\lambda}$.

But defining the length connection requires the further condition that the congruent displacement of a vector does not alter its length. In spite of its apparently tautological flavour, this clause avers that a gauge-system may be found for which $\delta \lambda=0$ when there is congruent displacement. Weyl then infers that the multiplying factor $d \phi$ being a linear differential form

$$
d \phi=\sum_{i} \phi_{i} d x_{i}
$$

is a necessary and sufficient condition for making $d \phi$, and hence $d l$, vanish. Assuming that the $\phi_{i}$ in $d \phi=\phi_{i} d x_{i}$ are given numbers at $P$, since $d \bar{\phi}=d \phi-\frac{d \lambda}{\lambda}$, it suffices to choose the gauge system $\lambda$ for which $d \phi=\frac{d \lambda}{\lambda}$, and therefore $\sum_{i} \phi_{i} d x_{i}=d(\log \lambda)$, holds. The linear differential form then expresses the property that the factor of proportionality $d \phi$ does depend neither on the length $l$ nor on the direction of the displaced vector, but only on the infinitesimal displacements $d x_{i}$, since four coefficients $\phi_{i}$ are attached to every point of the space-time manifold and may vary from one point to another.

The metrical structure of the space-time manifold may then be characterized, according to Weyl, by two fundamental differential forms, namely one quadratic form $g_{i j} d x_{i} d x_{j}$ and one linear form $\phi_{i} d x_{i}$, so that the ten potentials $g_{i j}$ and the four coefficients $\phi_{i}$ are not only invariant under coordinate transformations but also transform according to the following gauge transformation rule for the length connection

$$
\bar{g}_{i j}=\lambda^{2} g_{i j}, \quad \bar{\phi}_{i}=\phi_{i}-\frac{1}{\lambda} \frac{\partial \lambda}{\partial x_{i}} .
$$

Weyl then envisages a conformal structure for which the ratio of the lengths of any two vectors at the same point and the angle between them are determined, but not the length of the vectors themselves, so that the gauge can be chosen arbitrarily at each point provided it is smooth. Since it is to be considered up to conformal equivalence, the metric no longer determines a single affine connection but a whole equivalence class thereof ${ }^{99}$.

\footnotetext{
${ }^{98}$ Cf. $[45, \S 16]$

$99[39$, p. 209] for the details.
} 
Therefore, as stressed in [35, p. 153], such a weakening of the metrical structure requires a further property - the "fundamental theorem of infinitesimal geometry", as Weyl calls it - which states that the affine connection can be defined in one and only one way so that parallel displacement does not alter the length of the displaced vector, and then can be made univocally compatible with the length connection.

As is well known, Weyl's main step consisted in identifying the $\phi_{i}$ coefficients of the linear form associated with the metric through the concept of gauging with the electromagnetic potentials ${ }^{100}$, thus aiming at unifying the laws of gravitation and the laws of electromagnetism within a common geometrical framework. Since transporting a vector from a point $P$ of the space-time manifold along a closed path generally modifies both direction and length, Weyl's geometrical programme roughly boils down to jointly ascribing the non-integrability of direction to the gravitational field and the non-integrability of length to the electromagnetic field, assuming that objective features of the world can thus be expressed by measurements owing to the continuous variation which affects a vector when transferred along a closed path.

\subsection{Shifting to the affine field}

Eddington's criticism of Weyl's theory mainly focused on the hybrid character that the notion of a gauge-system would inevitably be endowed with, being supposedly partly conventional and partly physical. Though, as explained in [35, p. 224], Weyl claimed that the comparison of the lengths of point-bound vectors pointing in different directions be immediately given by an eidetic intuition through the imaginative rotation of the first vector about its location point, in an allegedly homogeneous space, until it coincides with the second vector, Eddington could not make sense of Weyl's phenomenological account unless as relying implicitly on optical appliances. Therefore, according to Eddington's reading of Weyl's theory, whereas one can compare lengths in different directions but at the same point by experimental methods, lengths at different points cannot be compared by physical transfer of clocks and rods and require gauge conventions. More generally Eddington incriminated Weyl's original presentation of his theory as involving some sort of "essential tension between an a priori epistemological principle of relativity of magnitude and the existence of a natural gauge of the world testified in actual measurements with rods and clocks"101. Eddington's way out then consisted in demarcating the "world geometry", which he conceived only as a "graphical representation" of the physical world, namely as a mere conventional, though as adequate as possible, geometrizing which as such is to be thought of as devoid of any assumption regarding the ultimate nature of things, on the one hand, from the "natural geometry" built out of actual measurements on the other hand, which he discarded at the outset as hybrid and would only reconstruct at a later stage owing to identifications. Representing physical quantities in a graphical way by geometrical magnitudes not only affords perspicuity by allowing to "set before ourselves a mass of information in such a way that the eye can take it in at a glance" 102 , but also explanatory power by making the whole range of geometrical notions available for extensive search and unpacking of the informative content thus encapsulated.

\footnotetext{
${ }^{100}$ Cf. [35, p. 158] for the details

101 [35, p. 225].

102 [12, p. 196].
} 
It is therefore reasonable to seek enlightenment by giving a graphical representation to all the physical quantities with which we have to deal. In this way physics becomes geometrized. But graphical representation does not assume any hypothesis as to the ultimate nature of the quantities represented. The possibility of representing the whole world of physics in a unified geometrical representation is a test not of the nature of the world but of the ingenuity of the mathematician. ${ }^{103}$

However, enhanced insight should not overshadow the fact that the geometrical space in which all relevant physical quantities are represented is to be thought of as "some new conceptual space - not actual space" 104 . Though it would later happen to spur Einstein's quest for a geometrical unified field theory ${ }^{105}$, Eddington's highly original reconstruction of physics from the postulation of a metric independent affine connection was thus meant at the outset to instantiate a notion of 'geometrization' which would neither remove, nor add, anything from, or to, Einstein's theory, but aimed at a better understanding of the known laws of physics. Technically, Eddington disentangles the affine connection $\Gamma_{i j}^{k}$ from the underlying metric tensor $g_{\mu \nu}$, posits it as a starting point, and then proceeds to develop the whole scheme of relativity theory out of the single notion of infinitesimal parallel displacement in addition to the assumption of an appropriate topology for the four-dimensional manifold. Accordingly a displacement which is to be conceived as a certain relation of extension between two nearby points is not to be thought of as a vector since, strictly speaking, it ought to be endowed with no length at all, inasmuch as no such thing as a metric is defined yet. Supposing then an infinitesimal displacement $A^{\mu}$ at the point $P$ of coordinates $x_{\mu}$, one would wish to select the one displacement $A^{\mu}+d A^{\mu}$ which has "some kind of equivalence" 106 to the displacement $A^{\mu}$ out of the whole collection of displacements bound to a nearby point $P^{\prime}$ of coordinates $x_{\mu}+d x_{\mu}$. Though the nature of this equivalence is left undefined and "need $(s)$ not be determinate observationally", Eddington assumes its having "some significance in regard to the ultimate structure of world"107. Since the most general expression for the continuous change of $A^{\mu}$ in the parallel transference from $P$ to $P^{\prime}$ is of the form

$$
d A^{\mu}=-\Gamma_{\nu \alpha}^{\mu} A^{\alpha} d x_{\nu}
$$

Eddington reversely postulates the $\Gamma_{\nu \alpha}^{\mu}$ as mere arbitrary coefficients and assigns them the task to settle the equivalence between nearby parallel displacements, while requiring that they fulfil one fundamental symmetry condition

$$
\Gamma_{\nu \alpha}^{\mu}=\Gamma_{\alpha \nu}^{\mu}
$$

resulting from the axiomatically posited parallelogram law which states that if a displacement $A B$ is equivalent to $C D$, then $A C$ is equivalent to $B D$. Therefore are these $\Gamma_{\nu \alpha}^{\mu}$ coefficients fitted to provide the building blocks out of which physics is to be reconstructed.

\footnotetext{
103 [12, p. 196].

$104[12$, p. 197]

${ }^{105}$ Cf. [35, ch. 8] and [21, 44.3 .2$]$ for a precise account of the interplay between Eddington and Einstein and their divergence about the meaning of affine connections and geometrization.

106 [12, p. 213].

$107[12$, p. 213].
} 
World-building then parallels the traditional derivation of the main tensors of Riemannian geometry by replacing them with constructs - named "in-tensors" in Eddingtonian parlance, though now better identified as scale covariant tensors, and marked by a prefixed asterisk - which not only possess the same properties than their Riemannian counterparts with regard to transformations of coordinates but are also unaffected by gauge transformations, which Eddington formulates as follows by giving them a slight twist of his own ${ }^{108}$ :

$$
\begin{array}{rlrl}
g_{\mu \nu}^{\prime} & =\lambda^{2} g_{\mu \nu} \\
\kappa_{\mu}^{\prime}=\kappa_{\mu}+\frac{\partial \phi}{\partial x_{\mu}}, & \phi & =\log \lambda .
\end{array}
$$

However, since any reference to the metrical tensor is to be dispensed with at the outset, one should proceed exclusively from the affine connection. Hence, for instance, according to the usual mode of derivation of the Riemann-Christoffel tensor, the total variation $\delta A^{\mu}$ which supposedly affects a given displacement $A^{\mu}$ subjected to parallel transference round a small circuit $C$, can be couched by means of (11) in terms of the coefficients $\Gamma_{\nu \alpha}^{\mu}$, as follows

$$
\delta A^{\mu}=\oint_{C} \frac{\partial A^{\mu}}{\partial x_{\nu}} d x_{\nu}=-\oint_{C} \Gamma_{\nu \alpha}^{\mu} A^{\alpha} d x_{\nu}
$$

and thus by converting the contour integral into a surface integral owing to Stokes' theorem, Eddington extracts out of it what he calls the generalized Riemann-Christoffel tensor

$$
{ }^{*} B_{\mu \nu \sigma}^{\epsilon}=-\frac{\partial}{\partial x_{\sigma}} \Gamma_{\nu \mu}^{\epsilon}+\frac{\partial}{\partial x_{\nu}} \Gamma_{\sigma \mu}^{\epsilon}+\Gamma_{\nu \alpha}^{\epsilon} \Gamma_{\sigma \mu}^{\alpha}-\Gamma_{\sigma \alpha}^{\epsilon} \Gamma_{\nu \mu}^{\alpha}
$$

which mirrors exactly its Riemannian analogue, except for the henceforth metric independent status of the $\Gamma_{\nu \mu}^{\epsilon}$. In addition to ${ }^{*} B_{\mu \nu \sigma}^{\epsilon}$, another scale covariant tensor - or another "in-tensor" in Eddington's terminology - is obtained by contraction of the former, namely ${ }^{*} G_{\mu \nu}={ }^{*} B_{\mu \nu \sigma}^{\sigma}$. Both ${ }^{*} B_{\mu \nu \sigma}^{\epsilon}$ and ${ }^{*} G_{\mu \nu}$ then play a key role in Eddington's theory inasmuch as they are deemed to be "the most fundamental measures of the intrinsic structure of the world" and to "take precedence over the $g_{\mu \nu}$ which are only found at a later stage of our theory" ${ }^{109}$. In a nutshell, as Eddington's inference roughly goes, ${ }^{*} B_{\mu \nu \sigma}^{\epsilon}$ gives rise to ${ }^{*} G_{\mu \nu}$ which breaks up into a symmetrical part, $R_{\mu \nu}$, and an antisymmetrical part, $F_{\mu \nu}$,

$$
{ }^{*} G_{\mu \nu}=R_{\mu \nu}+F_{\mu \nu}
$$

so that by identifying $R_{\mu \nu}$ with the gravitational potential or the metric and $F_{\mu \nu}$ with the electromagnetic force, one obtains a bifurcation of geometry and electrodynamics out of a single world-geometric scale covariant tensor

$$
{ }^{*} G_{\mu \nu}=g_{\mu \nu}+F_{\mu \nu}
$$

\footnotetext{
${ }^{108}$ Note that Eddington's notation is consistent with Weyl's, inasmuch as what Weyl denoted $\bar{\phi}_{i}=$ $\phi_{i}-\frac{1}{\lambda} \frac{\partial \lambda}{\partial x_{i}}$ (cf. above) corresponds now to Eddington's $\kappa_{\mu}^{\prime}=\kappa_{\mu}+\frac{\partial \phi}{\partial x_{\mu}}$ so that, since $d \phi=d(\log \lambda)=\frac{d \lambda}{\lambda}$, it is clear by identification that $\kappa_{\mu}$ corresponds to $-\phi_{\mu}$ (in Weyl's notation), or in other words that Eddington flipped sign in the scale connection.

$109[12$, p. 215$]$
} 
As pointed out by [21], "Eddington's main goal in this paper [viz. [11]] was to include matter as an inherent geometrical structure: "What we have sought is not the geometry of actual space and time, but the geometry of the world-structure which is the common basis of space and time and things "([11, p. 121]). By "things" he meant : (1) the energy-momentum tensor of matter, i.e. the electromagnetic field, (2) the tensor of the electromagnetic field, and (3) the electric charge-and-current vector. His aim was reached in the sense that all three quantities were fixed entirely by the connection; they could no longer be given from the outside." 110 Still the main feature of Eddington's line of thought lies in his outright conventional setting of the so-called "natural gauge of the world". Since the introduction of an arbitrary metric $g_{\mu \nu}$ is required in order to assign lengths to displacements $A^{\mu}$ by simply stipulating that $l^{2}=g_{\mu \nu} A^{\mu} A^{\nu}$, Eddington avers that the $g_{\mu \nu}$ must be so chosen as to make the lengths of displacements agree with the lengths resulting from measurements, which can only be guaranteed if "the tensor $g_{\mu \nu}$ which defines the natural gauge is not extraneous, but is a tensor already contained in the world-geometry" 111 , namely ${ }^{*} G_{\mu \nu}$. Time is ripe then for fixing the natural gauge, since, assuming $l^{2}={ }^{*} G_{\mu \nu} A^{\mu} A^{\nu}$, one obtains $l^{2}=R_{\mu \nu} A^{\mu} A^{\nu}$ by simply cancelling the antisymmetrical part of ${ }^{*} G_{\mu \nu}$, so that the gauging equation $R_{\mu \nu}=\lambda g_{\mu \nu}$ obviously recommends itself, while by the same stroke, so to speak, matter is identified with $R_{\mu \nu}-\frac{1}{2} g_{\mu \nu} R$, as in Einstein's theory. Therefore, as [35] stresses it, "the fundamental field equations of gravitation and electromagnetism already appear through mathematical identification with tensors of world-geometry, and so no appeal need be made to a special action principle to derive them"112. Still, as will be seen, variational differentiation remains operative in Eddington's full-grown world-geometrical framework, though performing a theoretical function that would henceforth be somewhat deflected from what it used to be at previous stages.

\section{3. "Hamiltonian differentiation" at large}

From the vantage point of graphical representation, Eddington could then reassess the various available action principles that lend themselves to the derivation of the fundamental laws of nature, by transposing them in affine field theoretic terms. Since, as seen earlier, action is traditionally represented by $\mathbf{A}=\int G \cdot \sqrt{-g} d \tau$, where $G$ is the scalar curvature, the straightest way, following Hilbert's action principle, would have been seemingly to consider the corresponding generalized form ${ }^{*} G \sqrt{-g}$ as the appropriate option, if only it had been a scale gauge invariant density, or, as Eddington puts it, an "in-invariant density", but it is not. When gauge transformations are taken into account, usual Riemannian invariants may indeed happen to undergo multiplication by a power of the gauge factor $\lambda$ and so turn out to be scale covariant quantities with weight, or, as Eddington dubs them, "co-invariants"113. As it is the case with * $G \sqrt{-g}$, a natural alternative would then be to shift to ${ }^{*} G^{2} \sqrt{-g}$, thus leading to Weyl's action principle ${ }^{114}$.

\footnotetext{
$110[21$, p. 48$]$

$111[12$, p. 219$]$

$112[35$, p. 231], my emphasis.

113 Eddington's notions of "co-tensors" and "co-invariants" refer indeed in Weyl's parlance to the notion of weight of a tensor, cf. [35, p. 160] for details.

${ }^{114}$ On Weyl's action principle, cf. [35, p. 166] for details
} 
However, from the broader perspective adopted by Eddington ${ }^{115}$, though there are no scale gauge invariants, or "in-invariants" as he says, that are function of the $g_{\mu \nu}$ and the $\kappa_{\mu}$, scale gauge invariant densities of the form $\mathfrak{A}=A \sqrt{-g}$ may easily be found. Inasmuch as $\sqrt{-g}$ becomes multiplied by $\lambda^{4}$ when gauge transformation is performed, it indeed suffices to complement it with any scale covariant quantity which would be multiplied by $\lambda^{-4}$, to obtain a whole range of in-invariant densities, as for instance

$$
\left({ }^{*} G\right)^{2} \sqrt{-g} ; \quad{ }^{*} G_{\mu \nu} \cdot{ }^{*} G^{\mu \nu} \sqrt{-g} ; \quad{ }^{*} B_{\mu \nu \sigma}^{\epsilon} \cdot{ }^{*} B_{\epsilon}^{\mu \nu \sigma} \sqrt{-g} ; \quad F_{\mu \nu} F^{\mu \nu} \sqrt{-g} .
$$

Assuming then that $\mathfrak{A}=A \sqrt{-g}$ is a scale gauge invariant density, either picked out of these or obtained by linear combination from these, the integral $\int \mathfrak{A} d \tau$ taken over a fourdimensional region of the space-time manifold would be invariant under coordinate- and gauge-transformations, and therefore would denote an absolute property of the region itself. Though Weyl thought it defendable to derive from this fact an argument in favour of the four-dimensional character of the world, since the Maxwell action, viz. $\int F_{\mu \nu} F^{\mu \nu} \sqrt{-g} d \tau$, which he deemed an essential ingredient of any action principle, would cease to be a pure number in any other number of dimensions ${ }^{116}$, Eddington dismissed all concerns pertaining to natural geometry and envisaged the whole question of action principles from a mere structural point of view.

Couched in Eddington's affine field theoretic terms, Weyl's action principle corresponds to the following action density

$$
A \sqrt{-g}=\left({ }^{*} G^{2}-\alpha F_{\mu \nu} F^{\mu \nu}\right) \sqrt{-g}
$$

where $\alpha$ is a numerical constant. Whereas Weyl considered his own action principle as "not [necessarily] realized in nature exactly in this form" ${ }^{117}$, nevertheless, at least as Eddington reconstructed his way of thinking, he made the hypothesis that it would still comply with the principle of stationary action for all variations $\delta g_{\mu \nu}, \delta \kappa_{\mu}$ vanishing at the boundary of the four-dimensional region, so that the corresponding variational derivatives would themselves vanish

$$
\frac{\mathbf{h} A}{\mathbf{h} g_{\mu \nu}}=0, \quad \frac{\mathbf{h} A}{\mathbf{h} \kappa_{\mu}}=0 .
$$

But this time, as a result of the cautious admission of an action principle which Eddington deemed "obviously speculative" ${ }^{118}$, the vanishing of the variational derivatives permits no more of any straightforward realist reading. Notwithstanding, "the procedure is instructive as showing the kind of unifying principle that is aimed at according to one school of thought" 119 , since equating both variational derivatives to zero allows one to extract from them some information which may shed light on the way constituents combine in the making of the action-density. Though Weyl was credited for having made "an undoubted advance towards the truth" when shifting from ${ }^{*} G \sqrt{-g}$ to $* G^{2} \sqrt{-g}$ and thus making a cosmological curvature term appear, Eddington's criticism nevertheless focuses on the artificiality of his action principle.

\footnotetext{
${ }^{115}$ Cf. [12, § 88].

${ }^{116}$ Cf. [35, p. 160] for a detailed account.

$117[12$, p. 210]

$118[12$, p. 212$]$

119 [12, p. 210]
} 
But the connection [between the gravitational and the electromagnetic component $]$ though reduced to simpler terms is not in any way explained by Weyl's action principle. It is obvious that his action as it stands has no deep significance; it is a mere stringing together of two in-invariants of different forms. To subtract $F_{\mu \nu} F^{\mu \nu}$ from ${ }^{*} G^{2}$ is a fantastic procedure which has no more theoretical justification than subtracting $E_{\mu}^{\nu}[$ i.e. the electromagnetic tensor $]$ from $E_{\mu}^{\nu}[$ i.e. the energy tensor of matter]. At the most, we can only regard the assumed form of action $\mathbf{A}$ as a step towards some more natural combination of electromagnetic and gravitational variables. $^{120}$

Therefore variational differentiation is to be thought of as a heuristic device which may help to achieve a better understanding of the way gravitation and electromagnetism might be pieced together, whether it be used to denounce opaque combinations as in Weyl's assumption of a natural gauge or to gain enhanced insight by fathoming the hidden relations between the various components involved. Accordingly Eddington even widens the whole technique of it in the line of graphical representation, inasmuch as, while "this generalised graphical scheme may or may not be helpful to the progress of our knowledge; we attempt it in the hope that it will render the interconnection of electromagnetism and gravitational phenomena more intelligible" ${ }^{121}$. Since "Hamiltonian differentiation" at large requires that the whole range of significant variations be taken into account, Eddington now envisages any scalar density $\mathfrak{K}=K \sqrt{-g}$ built out of a scale gauge invariant $K$ as a function of $g_{\mu \nu}, F_{\mu \nu}, \kappa_{\mu}$, and their derivatives up to any order, so that the integral over a four-dimensional region

$$
\int \mathfrak{K} d \tau
$$

be an invariant. Eddington's idea is then to apply the method of variational differentiation which proved useful at earlier stages to get in tune with nature's own bearings, to world invariants containing electromagnetic variables. By partial integration, he obtains as previously

$$
\delta \int \mathfrak{K} d \tau=\int\left(\mathfrak{P}^{\mu \nu} \delta g_{\mu \nu}-\mathfrak{H}^{\mu \nu} \delta F_{\mu \nu}+\mathfrak{Q}^{\mu} \delta \kappa_{\mu}\right) d \tau
$$

for variations which vanish at the boundary of the region, assuming that, according to Eddington's notational conventions as set out in [12, p. 111], the quantities occurring in the above integral, namely $\mathfrak{P}^{\mu \nu}=P^{\mu \nu} \sqrt{-g}, \mathfrak{H}^{\mu \nu}=H^{\mu \nu} \sqrt{-g}$ and $\mathfrak{Q}^{\mu}=Q^{\mu} \sqrt{-g}$, respectively correspond to the following variational derivatives

$$
P^{\mu \nu}=\frac{\mathbf{h} K}{\mathbf{h} g_{\mu \nu}}, \quad H^{\mu \nu}=-\frac{\mathbf{h} K}{\mathbf{h} F_{\mu \nu}}, \quad Q^{\mu}=\frac{\mathbf{h} K}{\mathbf{h} \kappa_{\mu}} .
$$

By piecing together tensors built out of the three main variational derivatives above, Eddington then derives from appropriate assumptions the following mathematical identity

$$
P_{\mu \nu}^{\nu}=-F_{\mu \nu} H_{\sigma}^{\nu \sigma}-\frac{1}{2}\left(F_{\mu \nu} Q^{\nu}+\kappa_{\mu} Q_{\nu}^{\nu}\right)
$$

$120[12$, p. 212$]$.
$121[12$, p. 197$]$. 
which can be used to suggest partial identifications with physical magnitudes ${ }^{122}$ and may then help to point out a better fitted alternative scale gauge invariant $K$.

In considering the results of substituting a particular function for $K$, it has to be remembered that the equation (100.42) [viz. here (34)] is an identity. We shall not obtain from it any fresh law connecting $g_{\mu \nu}$ and $\kappa_{\mu}$. The final result after making the substitutions will probably be quite puerile and unworthy of the powerful general method employed. The interest lies not in the identity itself but in the general process of which it is a result. We have seen reason to believe that the process of Hamiltonian differentiation is naturally the process of creation of a perceptual world around us, so that in this investigation we are discovering the laws of physics by examining the mode in which the physical world is created. The identities expressing these laws may be trivial from the mathematical point of view when separated from the context; but the present mode of derivation gives the clue to their significance in our experience as fundamental laws of nature. ${ }^{123}$

At this stage, simplicity should complement perspicuity as a key criterion allowing to select the scale gauge invariant $K$. Whereas dismissing Weyl's action principle, Eddington then calls attention to a heretofore unnoticed regional in-invariant, namely

$$
\int \sqrt{-\left.\right|^{*} G_{\mu \nu} \mid} d \tau
$$

which is "not more irrational than the other in-invariants since these contain $\sqrt{-g}$ " 124 , while this one does not. Eddington names it the "generalised volume" since it is analogous to both the metrical volume and the electromagnetic volume of the region, though not endowed yet with a definitive physical interpretation. Provided that ${ }^{*} G_{\mu \nu}$ is the building material out of which the world is to be constructed, it is a natural step to look for the simplest scale gauge invariants that can be derived from it. Still the meaning of 'simple' is ambiguous, but, as Eddington suggests, one can 'take the order of simplicity to be the order in which the quantities appear in building the physical world from the material ${ }^{*} G_{\mu \nu}{ }^{\prime 125}$. In this sense, the generalised volume is simpler than the other scale gauge invariants, inasmuch as it can be built independently of the process of gauging as it contains nothing which depends on the $g_{\mu \nu}$ as fixed by that means. Though still remaining speculative, this proposal would nevertheless advance our knowledge, since "if this view is correct, that the invariants which give the ordinary equations adopted in physics are really approximations to more accurate expressions based on the generalised volume, it becomes possible to predict the second-order terms which are needed to complete the equations currently used" 126 .

\section{Concluding remarks : how cracks in world-building call for reverse struc- tural realism}

Eddington's original claim that "the investigation of the external world in physics is a quest for structure rather than substance" 127 stemmed from the idea that though

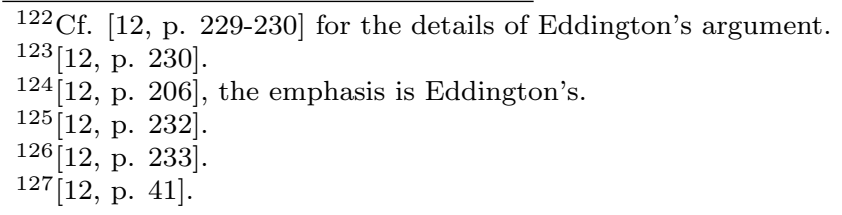


"anything permanent tends to become dignified with an attribute of substantiality"128, any such element of permanence is to be thought of as essentially contributed by the mind selecting the kind of pattern it is interested in spotting whatever Nature provides on its own. The mind's choosing a permanent pattern as the substance of the world thus boils down to the identification of matter with a suitable "substantial tensor" ${ }^{22}$, resulting in imposing laws onto the world. However, Eddington initially resisted the view of such "selective subjectivism" leading to completely resigning any attempt to grasp objectivity whatsoever.

Now it appears that a great number of the well-known laws of physics, mechanics and geometry are implicitly contained in this identification of substance. That is to say, these laws do not govern the course of events in the objective world, but are automatically imposed by the mind in selecting what it considers to be substance. They are identities in the definition of the geometrical character of the pattern which the mind hunts out. If all the discoveries of physics related to laws of this kind, we should be forced to admit that physics has nothing to contribute to the great question of how the world outside us is governed. I am not yet prepared to admit that. I think that we do, more especially in modern physics, encounter the genuine laws governing the external world, and are attempting - perhaps rather unsuccessfully - to grapple with them. But the great exact laws of gravitation, mechanics and electromagnetism, by which physics has won its high reputation as an exact science, all appear to belong to the other category; and, when these are set aside as irrelevant, our claim to have grasped the type of law, or even the meaning of law, prevailing in the world outside us is reduced to very modest proportions. ${ }^{130}$

Still, in the late twenties, Eddington would call for such genuine laws which, significantly enough, he calls "transcendental laws", by contrast with "identical laws" on the one hand, which are "obeyed as mathematical identities in virtue of the way in which the quantities obeying them are built"131, and "statistical laws" on the other hand, which reflect uniformity of averages, however uncertain may be the behaviour of the individuals composing the crowds whose behaviour proves steady and predictable.

If there are any genuine laws of control of the physical world, they must be sought in the third group - the transcendental laws. The transcendental laws comprise all those which have not become obvious identities implied in the scheme of worldbuilding. They are concerned with the particular behaviour of atoms, electrons and quanta - that is to say, the laws of atomicity of matter, electricity and action. We seem to be making some progress towards formulating them, but it is clear that the mind is having a much harder struggle to gain a rational conception of them than it had with the classical field laws. We have seen that the field laws, especially the laws of conservation, are indirectly imposed by the mind which has, so to speak, commanded a plan of world-building to satisfy them. It is a natural suggestion that the greater difficulty in elucidating the transcendental laws is due to the fact that we are no longer engaged in recovering from Nature what we have ourselves put in Nature, but are at last confronted with its own intrinsic system of government. But I scarcely know what to think. ... It may be that the laws of atomicity, like the

\footnotetext{
128 [13, p. 242].

129 [12, p. 120].

$130[10$, p. 421], the emphasis is mine.

131 [13, p. 244].
} 
laws of conservation, arise only in the presentation of the world to us and can be recognised as identities by some extension of the argument we have followed. But it is perhaps as likely that after we have cleared away all the superadded laws which arise solely in our mode of apprehension of the world about us, there will be left an external world developing under genuine laws of control. ${ }^{132}$

It should be noted that Eddington uses here the phrase "transcendental laws" as meaning laws that are beyond our grasping them as mathematical identities, though perhaps only temporarily, hence precisely not in the sense of "transcendental idealism" as referring to the conditions of possibility of our getting hold of an objective world constituted in experience. Therefore, though akin to a certain extent to transcendental idealism, Eddington's "selective subjectivism" should nevertheless be contrasted with this philosophical doctrine, inasmuch as allowing ways of approaching independent reality beyond mind's selected patterns. "Hamiltonian differentiation", at least at a first stage, was precisely intended to conjoin this concern for mind-independent objectivity with mind's "hunger for permanence" ${ }^{133}$. However, when hereafter shifting to affine field theory, Eddington would have to grant his affine field-based physics objective content by altogether different means since direct reference to the outside world through measurements had been jeopardised by the adoption of the affine connection as the fundamental quantity, as Pauli already pointed out to Eddington :

The quantities $\Gamma_{\nu \alpha}^{\mu}$ cannot be measured directly, but must be obtained from the directly measured quantities by complicated calculational operations. Nobody can determine empirically an affine connection for vectors at neighbouring points if he has not obtained the line element before. Therefore, unlike you and Einstein, I deem the mathematician's discovery of the possibility to found a geometry on an affine connection without a metric as meaningless for physics, in the first place. ${ }^{134}$

However, in Eddington's view, world-building was not to be expected to provide more than "a shadow performance of the drama enacted in the world of experience" 135 , viz. a graphical representation whose "success [consists] in the greatest contrast between the specialised properties of the completed structure [viz. the field laws] and the unspecialised nature of the basal material [viz. the 256 building blocks which, as explained below, result from mere structural weaving of relations and thus yield a local numerical measure of the world-structure]." 136 Now Eddington deemed his own world-building enterprise in the line of the method widely accepted among modern physicists, which, by making up things of familiar experience such as light and matter out of things "whose nature is left in suspense" ${ }^{137}$, eventually brought about a crisis when it came to be realized that "the linkage to familiar concepts should be through the advanced constructs of physics and not at the beginning of the alphabet" ${ }^{138}$. No complete explanation of anything physical is to be thought of as available whatsoever, insofar as no ultimate reduction of these abstract concepts in terms of the familiar ones is to be hoped for. Einstein's

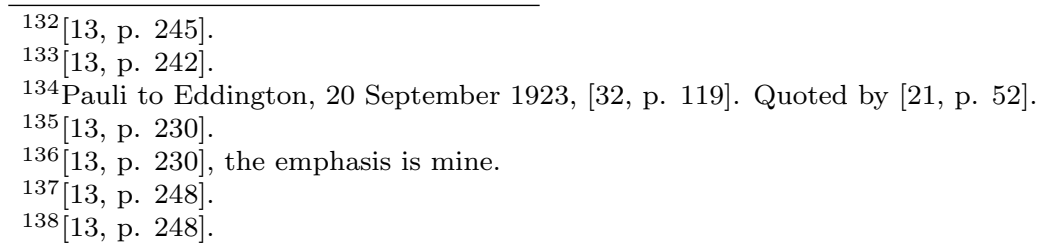


law for instance presents itself as a statement about certain qualities called potentials complying with certain differential equations. Still, even in this apparently unambiguous case, perplexities arise as soon as one pushes further by asking what are potentials. Eddington would thus later claim in his own peculiar entertaining way, that the method of physics involves a cyclic scheme akin to the well-known never-ending cumulative rhyme "The House that Jack built" 139 , inasmuch as the explanatory process would be doomed to go round and round endlessly, more or less as follows : this is potential, that was derived from the space-time interval, that was measured by scales and clocks, that were made of matter, that embodied mass, momentum and stress, that can be defined in terms of potentials, that were derived from the space-time interval, and so on and so forth, endlessly. Though one may be tempted to interrupt the cycle somewhere by saying that we know what matter is, at least as it occurs in everyday experience, Eddington emphasizes at this stage that the point of contact of the mind with the physical world is not very definite, disregarding his earlier attempts to determine it to a certain extent owing to variational derivatives. Since "the physical entities have a cyclic connection, and [since] whatever intrinsic nature we attribute to one of them runs as a background through the whole cycle" ${ }^{140}$, consciousness is then the only "touchstone of actuality" 141 owing to which the actual physical world can be discerned from all other mathematical worlds in which the same laws of nature hold. As he laid stress on "the potentiality of being known to the mind as a fundamental objective property of matter, giving it the status of actuality whether individual consciousness is taking note of it or not" ${ }^{142}$, Eddington then seemed to retreat to a rather coarse version of mind-stuff theory, while claiming structuralism as his professional view.

Insofar as our idea of substance "begins to fade when we analyze it" ${ }^{143}$, since concrete daily experienced matter eventually happens to be replaced by some symbolic counterpart instantiated in a suitable tensor, Eddington indeed envisages mind-stuff as a substratum that would underlie "mere connectivity of pointer-readings" ${ }^{44}$, thus providing world-building with a basis allowing to anchor somehow the scheme of physics in direct acquaintance.

To put the conclusion crudely - the stuff of the world is mind-stuff. As is often the way with crude statements, I shall have to explain that by "mind", I do not here exactly mean mind and by "stuff" I do not at all mean stuff. Still this is about as near as we can get to the idea in a simple phrase. The mind-stuff of the world is, of course, something more general than our own individual conscious minds; but we may think of its nature as not altogether foreign to the feelings in consciousness. ${ }^{145}$

Eddington's plunging into the "deep waters of philosophy" inasmuch as outlining such a tentative mind-stuff theory is less supported by some daring confidence in any alleged swimming capacities, as he himself put it, than it aims at trying "to show that the water is really deep" 146 . So as to convince physicists who might find difficult to admit that the

\footnotetext{
${ }^{139}$ Cf. [13, 261-263].

$140[13,268]$.

$141[13,266]$.

142 [13, 267], my emphasis.

143 [13, p. 273].

144 [13, p. 255].

145 [13, p. 276]

146 [13, p. 276].
} 
basal stuff out of which the world is to be built is of a mental nature, Eddington argues that (1) all our physical knowledge proceeds by inferences from the scarce islands of conscious awareness in our mental lives, that (2) consciousness is not so well demarcated and gradually merges into a whole range of less actual and more dispositional mental states, and eventually that (3) mind-stuff conceived of as the fundamental basso continuo underlying all such mental states appear as the best potential candidate so as to provide the intended basis for world-building.

We know that in the mind there are memories not in consciousness at the moment but capable of being summoned into consciousness. We are vaguely aware that things we cannot recall are lying somewhere about and may come into the mind at any moment. Consciousness is not sharply defined, but fades into subconsciousness; and beyond that we must postulate something indefinite but yet continuous with our mental nature. This I take to be world-stuff. We liken it to our conscious feelings because, now that we are convinced of the formal and symbolic character of the entities of physics, there is nothing else to liken it to. ${ }^{147}$

The rationale for such disconcerting mixture of structuralism and mind-stuff theory, rooted in the world-building enterprise and progressively matured into a philosophical stance by the end of the decade, is thus to be found in the urge for an overall scheme which would grant structural knowledge of the external world without renouncing to account for its having actual substance. Since Eddington's whole approach consisted, as previously shown, in starting with the affine connection $\Gamma_{\alpha \beta}^{\sigma}$ thought of as given independently from any underlying metric tensor $g_{\mu \nu}$, the main thrust of his later view would boil down to a purely structural reading of his earlier affine field theory in order to derive the whole of relativistic physics out of nothing but the fundamental relation of likeness of relations. Insofar as a structure can be thought of as a complex of relations and relata, Eddington would aim at getting rid of relata by framing an "interlocking of relations" which was meant to mimic ${ }^{148}$ the way the generalized Riemann-Christoffel tensor ${ }^{*} B_{\mu \nu \sigma}^{\epsilon}$ is built by unfolding its inner structure, inasmuch as this scale covariant tensor, or this "in-tensor" as Eddington calls it, was precisely intended to encapsulate "the most fundamental measure of the intrinsic structure of the world"149. Assuming first that the relata, which are presumably to be thought of as the point events of the space-time manifold, may be distinguished by four monomarks, that relations between relata can be compared and eventually that there is a relation of likeness between such relations, so that, when considering any relation between two given relata, one can pick out two other nearby relata which stand to one another in a like relation, the "ontological entwining of relations and relata" can thus be achieved, as stressed in [20, p. 233], by keeping track of the way one relation transforms into another around a closed path resulting in "a numerical measure of the structure surrounding the initial relatum" 150 . From such a "structural interlocking" of relations, the physical features of the world could then allegedly be obtained. By contracting the above original tensor of the fourth rank

\footnotetext{
$147[13$, p. 280].

${ }_{148}$ Compare for instance Einstein's geometric presentation of the standard derivation of the RiemannChristoffel tensor by means of Stokes' theorem, as given in [17, p. 75], with Eddington's diagrammatization of the so-called "structural interlocking of relations" in [14, p. 234].

$149[12$, p. 215]

$150[13$, p. 234$]$
} 
into one of the second rank, the number of relevant coefficients drops to 16, thus yielding 10 symmetrical coefficients on the one hand, corresponding to Einstein's potentials $g_{\mu \nu}$, from which space, time and "world-curvatures representing the mechanical properties of matter, viz. momentum, energy, stress, etc." $[13$, p. 236]. can be derived, and 6 antisymmetrical ones on the other hand, corresponding to the three components of the electric intensity and the three components of the magnetic force, from which electric and magnetic potential, electric charge and current, electromagnetic waves, etc. are obtained. Inasmuch as relying ultimately on the presumably primitive relation of likeness between relations, Eddington's sheerly structural account eventually gives way to mindstuff theory.

The only definition we can give of the aspect of the relations chosen for the criterion of likeness, is that it is the aspect which will ultimately be concerned in the getting into touch of mind with the physical world. But that is beyond the province of physics. (...) Let me confess at once that I do not know what I mean here by "very close together". As yet space and time have not been built. Perhaps we might say that only a few of the relata possess relations whose comparability to the first is definite, and take the definiteness of the comparability as the criterion of contiguity. I hardly know. The building at this point shows some cracks, but I think it should not be beyond the resources of the mathematical logician to cement them up. ${ }^{151}$

Eddington could not have alluded more transparently to Whitehead and Russell under this denomination of the presumably allied "mathematical logicians". As the cracks to be cemented up appear where physics and mind are supposed to meet, Eddington saw both Whitehead's ${ }^{152}$ and Russell's work ${ }^{153}$ on relativity theory as addressing, though from a different perspective, the same issue of linking physics with the world as given to mind in familiar experience. Insofar as claiming the fundamental uniformity of nature, Whitehead indeed dismissed the conception of the space-time manifold as made up of ultimately unanalyzable space-time points, hence the alleged "casual heterogeneity" 154 of spacetime relations in Einstein's theory, and owing to his method of extensive abstraction, he set out to build the space-time manifold out of a "continuum of events" so as to account for the required underlying basis of uniformity.

Nature presents itself to us as essentially a becoming, and any limited portion of nature which preserves most completely such concreteness as attaches to nature itself is also a becoming and is what I call an event. By this I do not mean a bare portion of space-time. Such a concept is a further abstraction. I mean a part of the becomingness of nature, coloured with all the hues of its content. Thus nature is a becomingness of events which are mutually significant so as to form a systematic structure. We express the character of the systematic structure of events in space and time. Thus space and time are abstractions from this structure. ${ }^{155}$

Following Whitehead, Russell would also make extensive use of mathematical logic so as to analyze the ultimate objects of physical theory as logical constructions out of events.

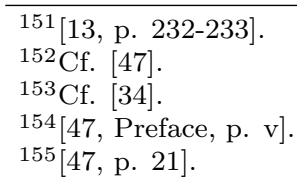


We shall find ... that the objects which are mathematically primitive in physics, such as electrons, protons, and points in space-time, are all logically complex structures composed of entities which are metaphysically more primitive, which may be conveniently called "events". It is a matter for mathematical logic to show how to construct, out of these, the objects required by the mathematical physicist. ${ }^{156}$

However, his logical analysis openly aimed at bridging the gap between physics on the one hand and the mass of percepts on the other hand, that Eddington's definition of matter by identification with a well-behaved tensor had inevitably brought about.

Approximately, matter as conceived by common sense is conserved; wherever it appears to be destroyed or created, we can find ways of explaining away this appearance. Hence, as an ideal suggested by empirical facts, we adopt the view that matter is indestructible. We then turn round, and beginning from the formula for interval we construct a mathematical quantity which is indestructible. This, we say, we shall call "matter"; and no harm comes of our doing so. But whenever we take a step of this sort, we widen the gulf between mathematical physics and observation, and increase the problem of building a bridge between them. ${ }^{157}$

Whereas he adopted a view of inferential knowledge according to which, as in Russell's theory of perception, we would reconstruct the world of physics out of the informative content entering our consciousness "in the form of messages transmitted along the nerves $(\ldots)$ as a paleontologist reconstructs an extinct monster from its footprint" 158 , Eddington considered Whitehead's approach as only apparently "diametrically opposed" to his, and inasmuch as it involved "a certain amount of working backwards", he envisaged it as a "tunnelling" from the opposite side of the mountain "to meet his less-philosophically minded colleagues" 159 . As variational derivatives eventually came to be divested of their original role as keeping the mind in step with nature, after graphical representation had tipped the scale of "Hamiltonian differentiation" in the sense of a heuristic device, Eddington would thus turn to mind-stuff to the same purpose, since "the word 'stuff' has reference to the function it has to perform as a basis of world-building and does not imply any modified view of its nature" 160 .

Acknowledgements. I thank Alexander Afriat, Marc Lachièze-Rey, Joël Merker and Jean-Jacques Szczeciniarz for fruitful discussions, and two anonymous referees for very helpful suggestions.

\section{References}

[1] Bach, R., 1921. Zur Weylschen Relativitätstheorie und der Weylschen Erweiterung des Krümmungstensorbegriffs. Mathematische Zeitschrift 9 (1-2), 110-135.

[2] Bicknell, G. V., 1974. Non-viability of Gravitational Theory Based on a Quadratic Lagrangian. Journal of Physics, A, Mathematical, Nuclear and General 7 (9), 1061-1069.

[3] Brading, K. A., Ryckman, T. A., 2008. Hilbert's Foundations of Physics : Gravitation and Electromagnetism Within the Axiomatic Method. Studies in History and Philosophy of Modern Physics 39 (1), 102-153.

\footnotetext{
$156[34$, p. 9-10].

$157[34$, p. 137]

$158[13$, p. 278$]$

$159[13$, p. 250$]$

$160[13$, p. 280$]$
} 
[4] Buchdahl, H. A., 1948. On Eddington's Higher-Order Equations of the Gravitational Field. Proceedings of the Edinburgh Mathematical Society 8, 89-94.

[5] Buchdahl, H. A., 1962. On the Gravitational Field Equations Arising From the Square of the Gaussian Curvature. Nuovo Cimento 23 (1), 141-157.

[6] Capozziello, S., Stabile, A., 2009. The Newtonian Limit of Metric Gravity Theories with Quadratic Lagrangians. Classical and Quantum Gravity 26, 1-22.

[7] Corry, L., 2004. David Hilbert and the Axiomatization of Physics (1898-1918). From Grundlagen der Geometrie to Grundlagen der Physik. Kluwer, Dordrecht-Boston-London.

[8] Eddington, A. S., 1920a. Space, Time and Gravitation. Cambridge University Press, Cambridge.

[9] Eddington, A. S., 1920b. The Meaning of Matter and the Laws of Nature According to the Theory of Relativity. Mind 29, 145-158.

[10] Eddington, A. S., 1920c. The Philosophical Aspect of the Theory of Relativity. Mind 29, 414-422.

[11] Eddington, A. S., 1921. A Generalisation of Weyl's Theory of the Electromagnetic and Gravitational Fields. Proc. R. Soc. London. Ser. A 99, 104-122.

[12] Eddington, A. S., 1923. The Mathematical Theory of Relativity. New edition, Cambridge University Press, 2007.

[13] Eddington, A. S., 1928. The Nature of the Physical world. Macmillan, New-York, Gifford lectures 1927.

[14] Eddington, A. S., 1939. The Philosophy of Physical science. Cambridge University Press, Cambridge, Tarner Lectures 1938

[15] Einstein, A., 1916. Hamiltonsches Prinzip und allgemeine Relativitätstheorie. Königlich Preussische Akademie der Wissenschaften (Berlin) Sitzungberichte, 1111-1116Reprinted in Collected Papers of Albert Einstein, Vol. 6, Doc. 41

[16] Einstein, A., March 1936. Physics and Reality. Journal of the Franklin Institute 221, 349-382, English translation by Jean Piccard.

[17] Einstein, A., 1946. The Meaning of Relativity, 2nd Edition. Princeton University Press, Princeton, NJ, Stafford Lectures, Princeton, 1921.

[18] Einstein, A., 1998. Collected Papers of Albert Einstein. Vol. 8A : The Berlin Years : Correspondence, 1914-1917. Vol. 8B : The Berlin Years : Correspondence, 1918. Princeton University Press, Princeton, r. Schumann et alii, eds.

[19] Einstein, A., Grossmann, M., 1913. Entwurf einer verallgemeinerten Relativitätstheorie und einer Theorie der Gravitation. Teubner, Leipzig, reprinted in Collected Papers of Albert Einstein, Vol. 4, Doc. 13.

[20] French, S., 2003. Scribbling on the Blank Sheet : Eddington's Structuralist Conception of Objects. Studies in History and Philosophy of Modern Physics 34, 227-259.

[21] Goenner, H., 2004. On the History of Unified Field Theories. Vol. 7. [Online article] : http://relativity.linvinreviews.org/lrr-2004-2.

[22] Havas, P., 1977. On Theories of Gravitation with Higher-Order Field Equations. General Relativity and Gravitation 8 (8), 631-645.

[23] Hilbert, D., 1915. Die Grundlagen der Physik. (Erste Mitteilung). Königliche Gesellschaft der Wissenschaften zu Göttingen. Mathematisch-physikalische Klasse. Nachrichten., 395-407.

[24] Hilbert, D., 1919/1920. Natur und Mathematisches Erkennen : Vorlesungen, gehalten 1919-1920 in Göttingen. Birkhäuser, Boston-Basel-Berlin, edited by David Rowe, 1992.

[25] Lanczos, C., 1938. A Remarkable Property of the Riemann-Christoffel Tensor in Four Dimensions. The Annals of Mathematics 39 (4), 842-850.

[26] Lanczos, C., 1969. Quadratic Action Principle of Relativity. Journal of Mathematical Physics 10 (6), $1057-1065$.

[27] Majer, U., Sauer, T., 2005. Hilbert's "World's Equations" and His Vision of a Unified ScienceIn Kox, A. J.., and Eisenstaedt, J.., The Universe of General Relativity, Birkhäuser, Boston-BaselBerlin.

[28] Mie, G., 1912. Grundlagen einer Theorie der Materie. Annalen der Physik37, 511-534; 39, 1-40; 40, $1-66$.

[29] Ohanian, H. C., October 1977. What is the Principle of Equivalence? American Journal of Physics 45 (10), 903-908.

[30] Pauli, W., June 1919a. Zur Theorie des Gravitation und Elektrizität von Hermann Weyl. Phys. Zeitschrift 20, 457-467.

[31] Pauli, W., November 1919b. Mercurperihelbewegung und Strahlenablenkung in Weyls Gravitationstheorie. Verh. Deutsch. Phys. Gesellsch. 21, 742-750.

[32] Pauli, W., 1979. Wolfgang Pauli. Wissenschaftlicher Briefwechsel mit Bohr, Einstein, Heisenberg, 
u. a. Bd. I : 1919-1929. Springer, New-York-Heidelberg-Berlin.

[33] Rowe, D., 2003. Hermann Weyl, The Reluctant Revolutionary. The Mathematical Intelligencer $25(2), 61-70$.

[34] Russell, B., 1927. The Analysis of Matter. New edition, Routledge, London \& New York, 1992.

[35] Ryckman, T., 2005. The Reign of Relativity. Philosophy in Physics 1915-1925. Oxford University Press, Oxford.

[36] Sauer, T., 1999. The Relativity of Discovery : Hilbert's First Note on the Foundations of Physics. Arch. Hist. Exact. Sci. 53, 529-575.

[37] Sauer, T., 2005. Einstein Equations and Hilbert Action : What is Missing on Page 8 of the Proofs for Hilbert's First Communication on the Foundations of Physics? Arch. Hist. Exact. Sci. 59, 577-590.

[38] Schilpp, P. A., 1949. Albert Einstein: Philosopher-Scientist. Northwestern University Press, Evanston, Illinois, reed. in The Library of Living Philosophers, vol. VII, Open Court, La Salle, Illinois, 1970

[39] Scholz, E., e., 2001. Hermann Weyl's Raum-Zeit-Materie and a General Introduction to His Scientific Work, DMV Seminar, Band 30 Edition. Birkhäuser, Basel-Boston-Berlin.

[40] Synge, J. L., 1971. Relativity : The General Theory. North-Holland, Amsterdam.

[41] Vizgin, V. P., 1994. Unified Field Theories in the first third of the 20th century. Birkhäuser, BaselBoston-Berlin.

[42] Weyl, H., 1918. Reine Infinitesimalgeometrie. Mathematische Zeitschrift 2, 384-411, republished in Ges. Ab., Vol. II, p. 1-28.

[43] Weyl, H., 1918/1921. Raum-Zeit-Materie, 4th Edition. Springer, Berlin, trans. by H. L. Brose as Space-Time-Matter, London, Meuthen. Repr. ed., New-York, Dover, 1953.

[44] Weyl, H., 1919. Eine neue Erweiterung der Relativitätstheorie. Annalen der Physik 59, 101-133, republished in Ges. Ab., Vol. II, p. 55-87.

[45] Weyl, H., 1921. Über die physikalischen Grundlagen der erweiteren Relativitätstheorie. Physikalische Zeitschrift 22, 473-480, republished in Ges. Ab., Vol. II, p. 229-236.

[46] Weyl, H., 1944. David Hilbert and his Mathematical Work. Bulletin of the American Mathematical Society $50,612-654$

[47] Whitehead, A. N., 1922. The Principle of Relativity with Applications to Physical Science. Cambridge University Press, Cambridge. 